\title{
Trichoderma reesei complete genome sequence, repeat-induced point mutation, and partitioning of CAZyme gene clusters
}

\author{
Wan-Chen Li ${ }^{1,2,3+}$, Chien-Hao Huang ${ }^{3,4 \dagger}$, Chia-Ling Chen ${ }^{3}$, Yu-Chien Chuang ${ }^{3}$, Shu-Yun Tung ${ }^{3}$ \\ and Ting-Fang Wang ${ }^{1,3^{*}}$ (])
}

\begin{abstract}
Background: Trichoderma reesei (Ascomycota, Pezizomycotina) QM6a is a model fungus for a broad spectrum of physiological phenomena, including plant cell wall degradation, industrial production of enzymes, light responses, conidiation, sexual development, polyketide biosynthesis, and plant-fungal interactions. The genomes of QM6a and its high enzyme-producing mutants have been sequenced by second-generation-sequencing methods and are publicly available from the Joint Genome Institute. While these genome sequences have offered useful information for genomic and transcriptomic studies, their limitations and especially their short read lengths make them poorly suited for some particular biological problems, including assembly, genome-wide determination of chromosome architecture, and genetic modification or engineering.

Results: We integrated Pacific Biosciences and Illumina sequencing platforms for the highest-quality genome assembly yet achieved, revealing seven telomere-to-telomere chromosomes (34,922,528 bp; 10877 genes) with 1630 newly predicted genes and $>1.5 \mathrm{Mb}$ of new sequences. Most new sequences are located on AT-rich blocks, including 7 centromeres, 14 subtelomeres, and 2329 interspersed AT-rich blocks. The seven QM6a centromeres separately consist of 24 conserved repeats and 37 putative centromere-encoded genes. These findings open up a new perspective for future centromere and chromosome architecture studies. Next, we demonstrate that sexual crossing readily induced cytosine-to-thymine point mutations on both tandem and unlinked duplicated sequences. We also show by bioinformatic analysis that T. reesei has evolved a robust repeat-induced point mutation (RIP) system to accumulate AT-rich sequences, with longer AT-rich blocks having more RIP mutations. The widespread distribution of AT-rich blocks correlates genome-wide partitions with gene clusters, explaining why clustering of genes has been reported to not influence gene expression in T. reesei.

Conclusion: Compartmentation of ancestral gene clusters by AT-rich blocks might promote flexibilities that are evolutionarily advantageous in this fungus' soil habitats and other natural environments. Our analyses, together with the complete genome sequence, provide a better blueprint for biotechnological and industrial applications.
\end{abstract}

Keywords: Trichoderma reesei, Complete genome sequence, Repeat-induced point mutation, AT-rich block, Centromere, Centromere-encoded gene, CAZyme gene cluster

\section{Background}

Trichoderma is a fungal genus found in many different natural habitats including-among others-mushroom

\footnotetext{
*Correspondence: tfwang@gate.sinica.edu

${ }^{\dagger}$ Wan-Chen Li, Chien-Hao Huang contributed equally to this work

${ }^{3}$ Institute of Molecular Biology, Academia Sinica, Taipei 115, Taiwan

Full list of author information is available at the end of the article
}

caps, rotting wood, and soil. Trichoderma reesei (syn. Hypocrea jecorina) is a widely used model organism for plant cell wall degradation and industrial enzyme production. The natural strain QM6a (ATCC13631) was first isolated from the Solomon Islands during the Second World War [1]. High enzyme producers (e.g., QM9414 and RUT-C30) were artificially generated from 
QM6a for industrial applications over the last 70 years [2-6].

Trichoderma reesei undergoes a heterothallic reproductive cycle and generates fruiting bodies (stromata) containing linear asci with 16 ascospores [7]. These 16 ascospores are generated via meiosis and two rounds of postmeiotic mitosis [8]. When placed under favorable conditions, ascospores germinate to form vegetative mycelia and produce asexual spores (i.e., conidia). Sexual development of the T. reesei CBS999.97 wild-isolate strain produced two haploid strains, CBS999.97(MAT1-1, F/X) and CBS999.97(MAT1-2, M/33). The two ancestral scaffolds (M and 33) in CBS999.97(MAT1-2, M/33) underwent an unequal translocation to form two new scaffolds (F and X) in CBS999.97(MAT1-1, F/X) [8]. Like CBS999.97(MAT1-2, M/33), QM6a has a MAT1-2 mating-type locus and two ancestral scaffolds $M$ and 33. Due to chromosome heterozygosity and meiotic recombination, sexual crossing of CBS999.97(MAT1-1, F/X) with CBS999.97(MAT1-2, M/33) or QM6a often (>90\%) generates segmentally aneuploid (SAN) progeny [8]. The CBS999.97 wild-isolate strain is also an excellent fungal model for light responses since light variation greatly affects its sexual development and conidiation $[7,9,10]$. Constant light promotes conidiation and completely inhibits stromata formation, whereas total darkness causes a slowdown of the growth of stromata [10].

The genomes of several Trichoderma species have been sequenced and are publicly available from the Joint Genome Institute (JGI) of the US Department of Energy. The QM6a-v2.0 draft genome (33.4 Mb) contains 87 scaffolds and 9129 predicted genes [11]. The RUTC30-v1.0 draft genome (32.7 Mb) has 182 scaffolds and 9852 predicted genes [12]. The genomes of Trichoderma atroviride and Trichoderma virens were thought to be larger than QM6a-v2.0, with sizes of 36.1 and $38.8 \mathrm{Mb}$, respectively, versus $34.1 \mathrm{Mb}$ for QM6a, both encoding more than 2000 additional predicted genes $[4,13]$. These genomes have been used to identify key genes involved in some important biological processes [4, 13-15], e.g., the transcriptional factors and transporters that control induction and expression of carbohydrate-active enzymes (CAZymes) and the plant cell wall degradation enzymes. Using QM6a as a reference, it has also been reported that QM9414 and RUT-C30 might carry multiple alternations, including rearrangements, point mutations, insertions, and deletions $[12,16]$. Recently, a group of Trichoderma researchers have collectively annotated and compared $\sim 30 \%$ genes in the JGI genomes of T. reesei, T. atroviride, and T. virens [4].

To reveal gene order and dynamic gene expression at the chromosome level, a genome-wide chromosome conformation capture method (referred herein as "HiC") had been applied to close the gaps between the QM6av2.0 scaffolds. The $\mathrm{HiC}$ draft genome revealed seven superscaffolds and four short contigs [17]. Druzhinina et al. [18] then applied the QM6a-HiC draft to annotate 9151 (not 9194) predicted genes. A third of the putative CAZyme genes occurred in loose clusters that also contained a high number of genes encoding small-secreted cysteine-rich proteins (SSCPs). Five CAZyme gene clusters are located close to chromosomal ends. These subtelomeric areas are also enriched in genes involved in conidiation, iron scavenging, and interactions with other fungi, such as secreted protease genes, amino acid transporter genes, gene clusters for polyketide synthases (PKS), non-ribosomal peptide synthase (NRPS), and PKS-NRPS fusion proteins. The QM6a-HiC annotation (http://trichocode.com/index.php/t-reesei) became publicly available in January, 2017 and the expectation values $(E)$ for the BLAST sequence alignments are not available, but claimed to be at least $<\mathrm{e}^{-75}[18]$.

Strictly speaking, the $\mathrm{HiC}$ draft genome is far from equivalent to a complete genome sequence. We noticed that both the QM6a-v2.0 and QM6a-HiC drafts lack several evolutionarily conserved genes that are ubiquitously expressed in almost all studied eukaryotic organisms, including the recA family protein Rad51 and the DNA repair protein Rad50. Therefore, we applied both second- and third-generation sequencing (SGS and TGS) platforms to resequence the QM6a genome. The single molecule real-time (SMRT) sequencing method developed by Pacific BioSciences (PacBio) offers much longer reads of up to $60 \mathrm{~kb}$ [19]. After error correction with short and high-quality Illumina MiSeq sequencing reads, the PacBio long reads were assembled into seven telomere-to-telomere chromosomes. Our high-quality genome sequence provides a large quantity of new information to facilitate functional and comparative studies of this industrially important workhorse fungus.

\section{Results \\ Resequencing the QM6a genome}

The QM6a genome was resequenced using seven SMRT cells on the PacBio RSII platform. Following extraction of reads with Trichoderma reesei-only sequences, we recovered the longest raw reads $(\geq 16 \mathrm{~kb})$ with up to $80 \times$ coverage, totaling $3,397,762,180 \mathrm{bp}$. The hierarchical genome assembly process program [20] was used to generate a preliminary PacBio draft with seven superscaffolds, a short unitig, and $1.8 \mathrm{~kb}$ of contaminating DNA (Additional file 1: Table A1). This short unitig was completely identical in nucleotide sequence to the QM9414 mitochondrial genome (42,139 bp; NC_003388.1) [21], indicating that the error rate for the preliminary PacBio draft was extremely low $(<0.0024 \%)$. 
For error correction, the Illumina MiSeq $300 \mathrm{bp}$ paired-end reads $(6.8 \mathrm{~Gb})$ were collected and trimmed. Reads $(67.85 \%)$ with a quality score threshold (Q) greater than 30 were retained (Additional file 1: Tables A2, A3). The final assembly data contain a circular mitochondrial genome (42,139 bp) and seven unitigs $(34,922,528 \mathrm{bp})$ (Additional file 1: Table A3). We highlight that there were no sequence ambiguities or unidentified bases (Ns) (Table 1). In contrast, the QM6a-v2.0 draft genome was $33,453,791 \mathrm{bp}$ and had 48,252 Ns, whereas the HiC draft was $33,395,328$ bp and had 42,879 Ns.

The seven superscaffolds closely match (if not being completely identical to) the full-length chromosomes because all of their termini capture typical telomeric sequences (i.e., TTAGGG at $3^{\prime}$-termini and the reverse complement CCCTAA at $5^{\prime}$-termini) [11] with up to 14 repeats (Additional file 1: Table A3). We categorized these telomere-to-telomere chromosomes with Roman numerals (ChI-ChVII), from largest to smallest. Genetically defined linkage groups were designated alphabetically (A-G), and linkage group arms are designated L for the short (left) arm at $5^{\prime}$ termini and $\mathrm{R}$ for the long (right) arm at $3^{\prime}$ termini (Fig. 1). The complete QM6a genome sequences have been submitted to NCBI (Accession Number CP016232-CP016238).

\section{The complete QM6a genome compared to two earlier draft genomes}

By mapping the MiSeq reads to the QM6a-v2.0 draft, we found that ten scaffolds in the QM6a-v2.0 draft were not genuine $T$. reesei sequences (Additional file 1: Tables A5). For this reason, 16 previously annotated genes (Additional file 1: Tables A6) in QM6a-v2.0 and QM6aHiC might not be authentic QM6a genes. In addition, there were numerous sequencing and assembly errors

\begin{tabular}{ll}
$\begin{array}{l}\text { Table } 1 \text { Summary of the QM6a genome sequencing } \\
\text { and assembly results }\end{array}$ & \\
\hline Total sequenced bases & $3,397,762,180 \mathrm{bp}$ \\
Max of all assembled unitigs & $6,835,650 \mathrm{bp}$ \\
$\mathrm{N}_{50}$ of all assembled unitigs & $5,311,312 \mathrm{bp}$ \\
Number reads & $263,312 \mathrm{bp}$ \\
$\mathrm{N}_{50}$ reads & $18,236 \mathrm{bp}$ \\
Phred quality score & 48.8 \\
Unitigs & Seven linear chromosomes \\
& Circular mitochondrial genome \\
Coverage & $81.4 \times(7$ chromosomes) \\
& $2437.6 \times$ (mitochondria) \\
Genome size & $34,922,528$ bp (7 chromosomes) \\
& $42,139 \mathrm{bp}$ (mitochondria) \\
Unidentified bases (N) & $0 \mathrm{bp}$ \\
GC content & $51.1 \%$ \\
Predicted genes & 10,877 (1630 new genes) \\
\hline
\end{tabular}

in QM6a-v2.0. The most prominent assembly error was the first and longest scaffold, wherein the $5^{\prime}$ portion $(2.46 \mathrm{Mb})$ was mapped to $\mathrm{ChV}$ and the $3^{\prime}$ portion $(\sim 1.79 \mathrm{Mb})$ to ChIV (Additional file 1: Table A7).

Our complete QM6a genome sequence also covers all seven superscaffolds and the four short contigs of QM6a-HiC [17]. QM6a-HiC contains a large quantity of sequence and/or assembly errors, particularly those sequences (e.g., telomeric repeats) close to both termini of the seven superscaffolds. The four short $\mathrm{HiC}$ contigs are all located at chromosome regions with low guanine (G)-cytosine (C) contents. HiC0 is located close to tel6L (the left telomere of $\mathrm{ChVI}$ ), $\mathrm{HiC} 1$ and $\mathrm{HiC} 2$ at cen3 (the centromere of ChIII), and HiC3 in an interspersed ATrich block on the right arm of ChVI. There were at least 18 inversion errors in the $\mathrm{HiC}$ draft genome (Fig. 1; Additional file 1: Table A8). Eight of these inversion errors could account for the failure to connect these four short contigs to the corresponding superscaffolds during the $\mathrm{HiC}$ experiments [17].

The HiC experiments also resulted in incorrect assembly at the rDNA locus. Using Southern hybridization, we confirmed that the right arm of ChVI harbors the large rDNA locus with nine tandem "head-to-tail" repeats. Each repeat contains an 18S-5.8S-26S rRNA gene cluster and a non-transcribed intergenic spacer (IGS) (Fig. 2; Additional file 1: Table A9). This result approaches the theoretical limit for mapping results using Illumina MiSeq short reads, i.e., $200-260 \times$ coverage at the rDNA locus versus $25-30 \times$ coverage along the entire chromosome (Fig. 3). It is worth noting that there are 175-200 copies of the large rDNA tandem repeats in Neurospora crassa [22].

Thus, the complete QM6a genome sequence has uncovered many sequencing and assembly errors in QM6a-v2.0 and QM6a-HiC. We suggest that caution should be exercised in applying $\mathrm{HiC}$ or the chromosome conformation capture method for gap-closing draft genome sequences produced by SGS technology and for other genomic analyses involved in AT-rich and repetitive sequences.

\section{QM6a compared to high enzyme producers}

The high-quality QM6a genome sequence we provide here is a better scaffold to order and orient contigs of other Trichoderma draft genomes previously generated by SGS technologies. It has been reported that there might be five or eleven potential translocations in RUTC30 $[12,16]$. A BLAST search revealed that there are only three promising translocations in RUT-C30: $\mathrm{ChV}$ to ChII (the first scaffold of RUT-C30), ChIII to ChI (the second scaffold of RUT-C30), and ChIII to ChIV (the fifth scaffold of RUT-C30). Theoretically, these three 


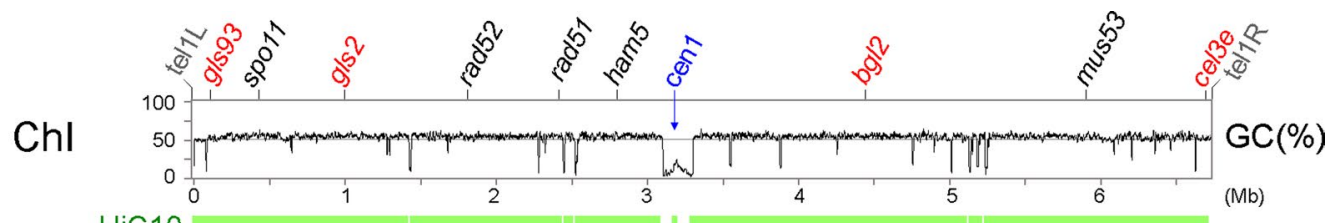

$\mathrm{HiC} 10$
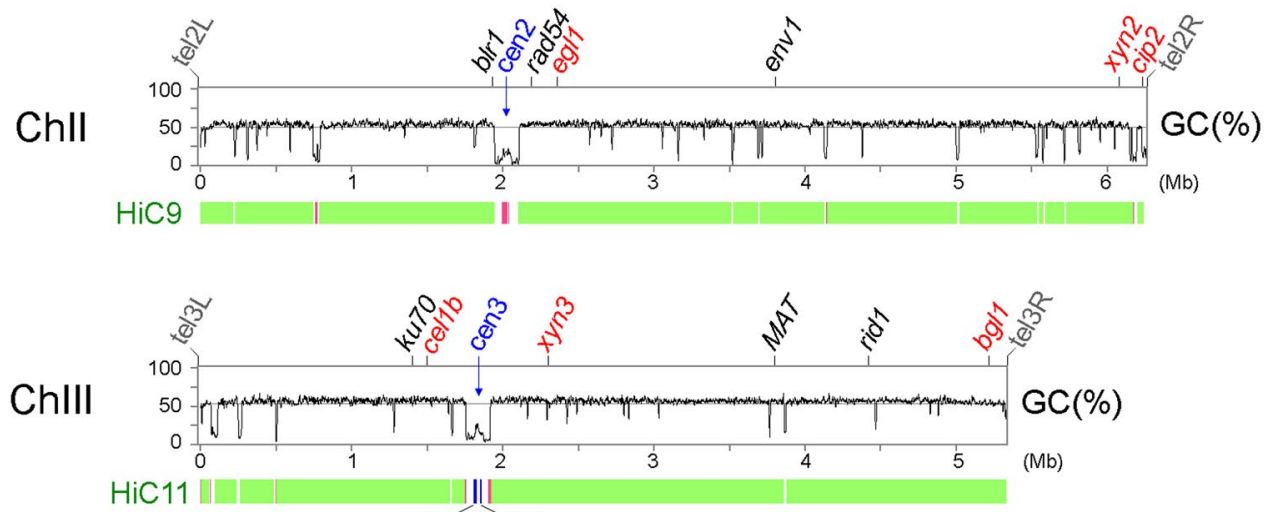

Hic2 $\mathrm{HiC} 1$
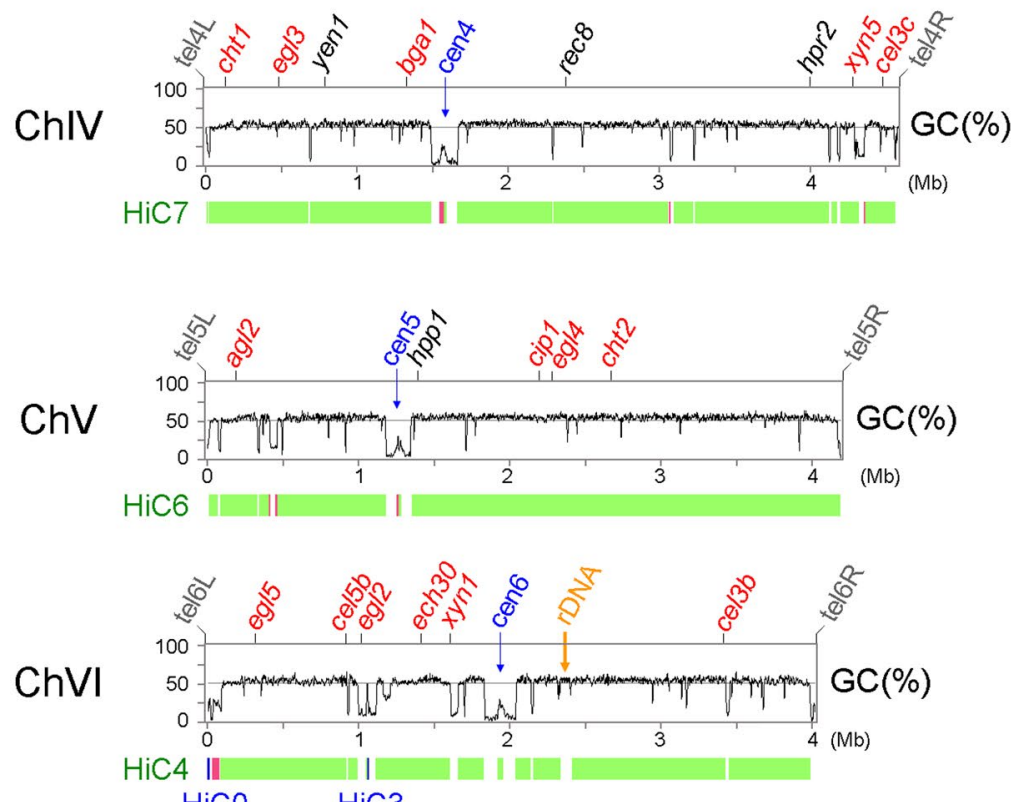

$\mathrm{HiCO} \quad \mathrm{HiC3}$

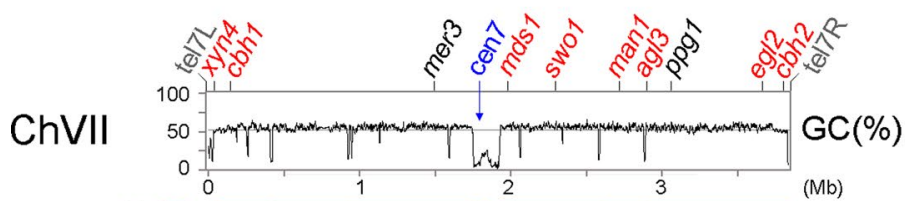

Hic5

Fig. 1 The complete QM6a genome compared to the HiC draft genome. The top tracks represent the graphs of GC contents (window size 5000 bp) of seven telomere-to-telomere chromosomes (Chl-ChVII). The seven centromeres (cen1-cen7; in blue) are located at the longest AT-rich blocks in each chromosome. The telomeres (tel) of the right (R) and left $(L)$ arms in each chromosome are indicated (in gray). The CAZyme genes (in red) and several genes involved in DNA repair, light response, and sexual development (in black) are indicated. The rDNA (18S-5.8S-26S) locus on ChVI is indicated in orange. The bottom tracks represent the seven superscaffolds (in green) and four short contigs (in blue) of the HiC draft genome. Chromosomal regions with incorrect orientation (i.e., inversion errors) are indicated in pink 
a

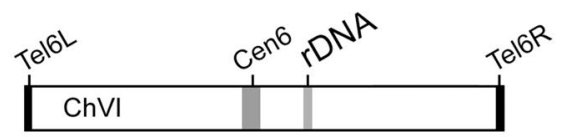

응

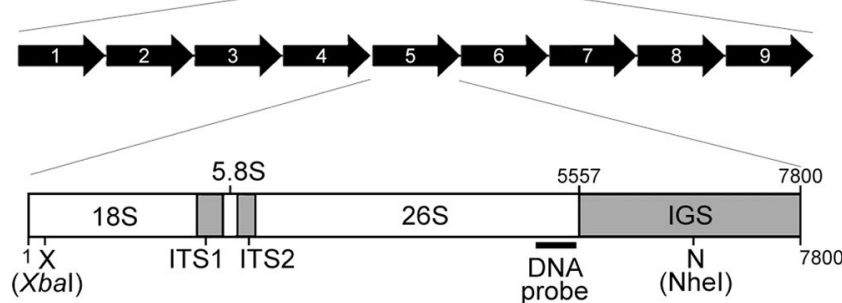

$$
\text { 오 }
$$

b

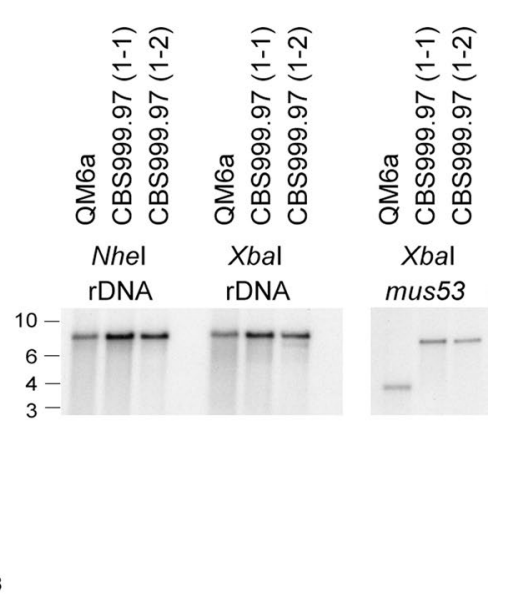

Fig. 2 The rDNA locus. a Organization of the rDNA locus. The top panel illustrates the nine tandem "head-to-tail" repeats revealed by the PacBio RSII platform. Each repeat contains an 18S-5.8S-26S rRNA gene cluster and a full-length non-transcribed intergenic spacer (IGS). The bottom panel shows the five repeats in the HiC draft genome. Each repeat has an 18S-5.8S-26S rRNA gene cluster and two truncated IGSs (IGS-5' $\Delta$ and IGS-3' $\Delta$ ). The locations of restriction enzymes Xbal (X) and Nhel (N) are indicted. b Southern hybridization. Genomic DNA (1 $\mu \mathrm{g})$ was isolated from three different wild-isolate strains: QM6a, CBS999.97(1-1) and CBS999.97(1-2). After digestion with Xbal (X) or Nhel (N), the genomic DNA was subjected to agarose gel electrophoresis, Southern blotting, and hybridization with a $28 \mathrm{~S}$ rDNA probe (a) or a mus53 probe (as the DNA loading control). The mus53 gene encodes the DNA ligase IV protein, and there is only one copy of the mus53 gene in the Trichoderma reesei genome [78]

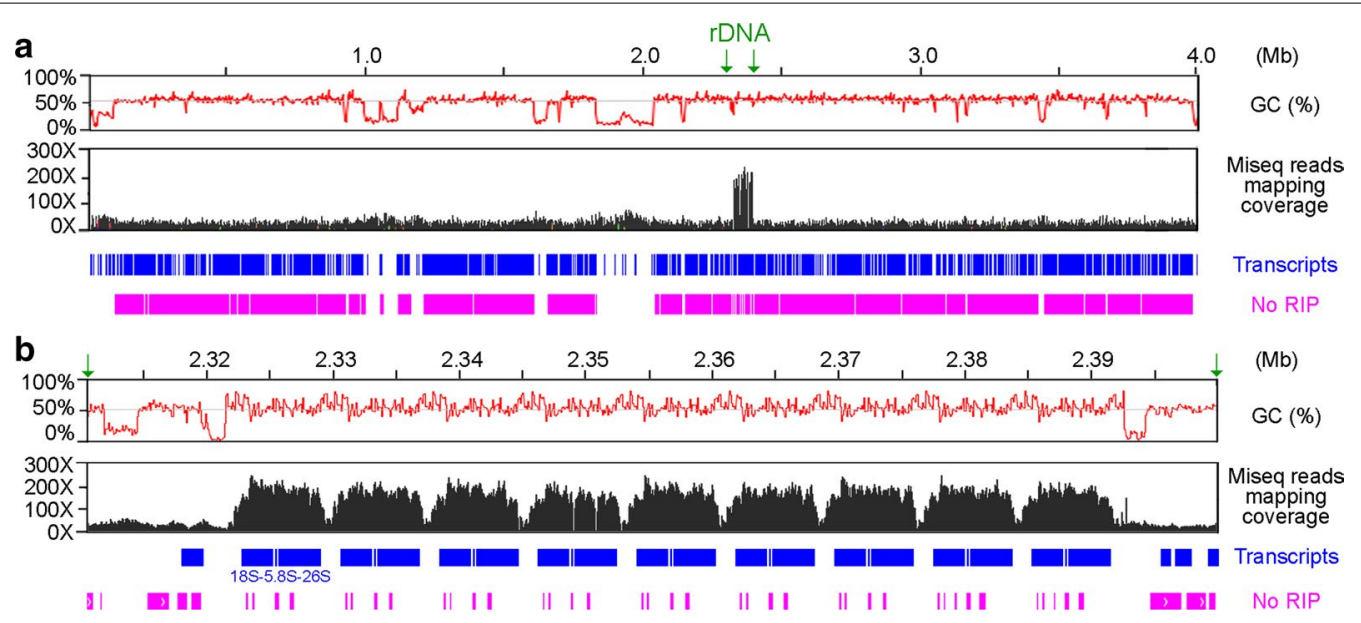

Fig. 3 The repetitive features of a representative QM6a chromosome (ChIV). The top tracks represent the graphs of GC contents (in red; window size 100 bp), the mapping coverage of Illumina MiSeq reads (in black), predicted genes (in blue), and the sequences not affected by RIP (No RIP; in pink) along the entire ChIV (a) and the rDNA locus $(\mathbf{b})$. The mapping coverage of Illumina MiSeq reads for overall genomic DNA is $\times 25-30$, whereas that specifically for the rDNA locus is $\times 200-260$. The RIPCAL program was used to predict the sequences mutated by RIP using default settings, and the sequences not affected by RIP are shown (No RIP; in pink)

translocations in RUT-C30, together with the one translocation we identified in CBS999.97(MAT1-1, F/X), are sufficient to account for the large quantity of inviable SAN ascospores generated from sexually crossing
RUT-C30 with CBS999.97(MAT1-1, F/X) [8, 23]. There are also three short ectopic insertions in RUT-C30 including the second scaffold ( $34 \mathrm{bp}$ ), the fifth scaffold (231 bp), and the ninth scaffold (2655 bp) (Additional 
file 1: Table A10). These three ectopic insertions are more likely due to sequence duplication or assembly errors.

\section{Genome reannotation}

We applied four different approaches to genome reannotation ("Methods"), including the use of all $(28,748)$ T. reesei proteins from National Center for Biotechnology Information (NCBI), QM6a-v2.0, RUT-C30-v1.0, and two publicly available transcriptome datasets [24, 25]. We annotated 1630 newly predicted QM6a genes, including 70 tRNA genes and 23 5S-rDNA genes (Additional file 2: Tables B1, B2). The average length of all 10,876 QM6a genes is $1579 \mathrm{bp}$. Their average GC content $(56.5 \%)$ is $\sim 5.5 \%$ higher than that of the entire QM6a genome. Among 1515 new protein-encoding genes, 679 have been annotated in RUT-C30-v1.0. Most of them encode novel or hypothetical proteins, and only 120 and 285 newly predicted genes encode protein products that have homologs in Saccharomyces cerevisiae and $N$. crassa, respectively. It is worth noting that we annotated several essential or biologically important genes, including six DNA repair genes (rad50, sae2/com1, rad51, rad57, srs2, rrm3, and pif1), an essential component of sister chromatin cohesion complex (smc1), a key autophagy gene (atg11), two cell division cycle genes $(c d c 4$ and $c d c 15)$, and two mitochondrial genes $(\operatorname{sod} 2$ and $t o m 7$ ) (Additional file 1: Table A11, Additional file 2: Tables B2, B3). These evolutionarily conserved genes had never been annotated in QM6a-v2.0 or QM6a-HiC [4, $11,18]$. Since our annotation results are compared well with those annotated recently by Schmoll et al. [4] and Druzhinina [18] (Additional file 2), the complete genome sequence and set of genes we provide here can serve as a better guide for further experiments, especially for global approaches to evolution, biological functions, and industrial applications.

We categorized all QM6a genes with a non-italicized uppercase letter, a number, and a letter: $\operatorname{Tr}$ (for T. reesei); A, B to G (for chromosome I, II through VII); a number corresponding to the order of the transcripts (counting from the left telomere); and $\mathrm{W}$ or $\mathrm{C}$ to designate the Watson or Crick strand (the Watson strand is $5^{\prime} \rightarrow 3^{\prime}$ left telomere-to-right telomere); for example, the 100th gene from the left telomere of chromosome I is TrA0100C. For previously annotated QM6a and RUT-C30 genes [11, 12], their JGI's gene identity number and standard names are also provided along with the systematic names (Additional file 2).

\section{Comparative transcriptome analysis}

Next, we applied TopHat-a bioinformatic sequence analysis package tool-to map and count the SGS reads of all annotated genes and then to determine the values of transcripts per kilobase million (TPM) [26]. Compared to reads per kilobase million (RPKM) and fragments per kilobase million (FPKM), TPM is a stable and reliable RNA-seq expression unit across experiments [27]. All reads mapped to the rRNA and tRNA genes were excluded before calculating TPMs (Additional file 2: Table B1).

Our results confirmed those reported by [24] that 35 CAZyme genes and 27 non-CAZyme genes were highly induced ( $\geq 20$-fold) by straw substrate in QM6a (Additional file 3: Table C1). In addition, straw also upregulated ( $\geq 20$-fold) 210 previously annotated genes in QM6a-v2.0, including xylanase regulator 1 (xyr1; $\geq 100$ fold), 38 CAZyme genes, 1 NRPS gene, the mating-type gene mat1-2-1 [7], and hybrid-type peptide pheromone precursor 1 (hpp1) [28] (Additional file 3: Table C2). Many straw-induced genes in QM6a [24] were shown to be differentially regulated in response to cellulose and sophorose in QM9414 [25] (Additional file 3: Tables C1, C2).

Among the 1535 new protein-encoding genes, there are 39 straw-induced ( $\geq 20$-fold) genes in QM6a and 2 cellulose-induced genes ( $\geq 20$-fold) in QM9414 (Additional file 3: Table C3). One hundred and forty-four new QM6a genes are also significantly upregulated (5- to 20-fold) by straw (Additional file 3: Table C4). For example, $\operatorname{TrC} 1345 \mathrm{C}$, a new straw-induced gene, encodes the homolog of S. cerevisiae Cat8 zinc-cluster transcription factor. Cat8 is involved in gluconeogenesis, the glyoxylate cycle, and ethanol utilization, and it is necessary for derepression of a variety of genes under non-fermentative growth conditions (e.g., diauxic shift and sporulation) [29]. Further research on this transcriptional factor might help to enhance cellulolytic enzyme production. We also identified nine new genes with TPM values in QM6a that are much higher than those in QM9414 (Additional file 3: Table C5). These genes are likely mutated or even deleted in QM9414.

\section{Wide distribution of AT-rich blocks along seven QM6a chromosomes}

To establish the link between DNA sequence and chromatin architecture, the local GC contents along each chromosome were calculated using a $0.5 \mathrm{~kb}$ sliding window. We identified 2349 AT-rich chromosome blocks with GC contents $\geq 12$ and $\geq 6 \%$ lower than the average GC content of the all predicted genes (56.5\%) and the entire QM6a genome (51.1\%), respectively (Table 2).

The most prominent or longest AT-rich blocks in each chromosome are the centromeres, ranging from $162.5 \mathrm{~kb}$ (cen2) to $208.5 \mathrm{~kb}$ (cen6) (Fig. 4 and Additional file 3: Table A2). The longest AT-rich blocks in each $N$. crassa chromosome are also centromeres [30]. A BLAST search 


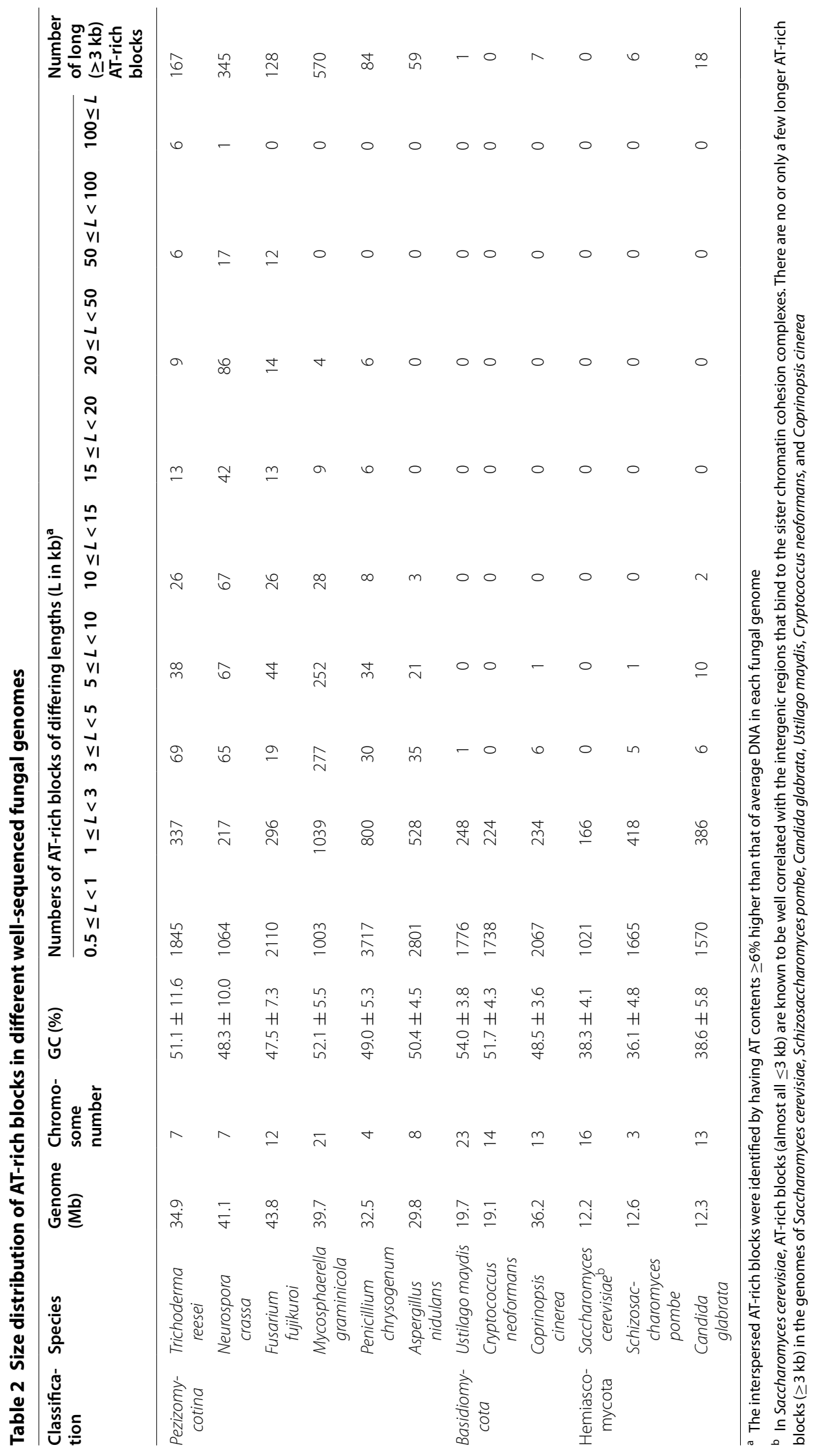




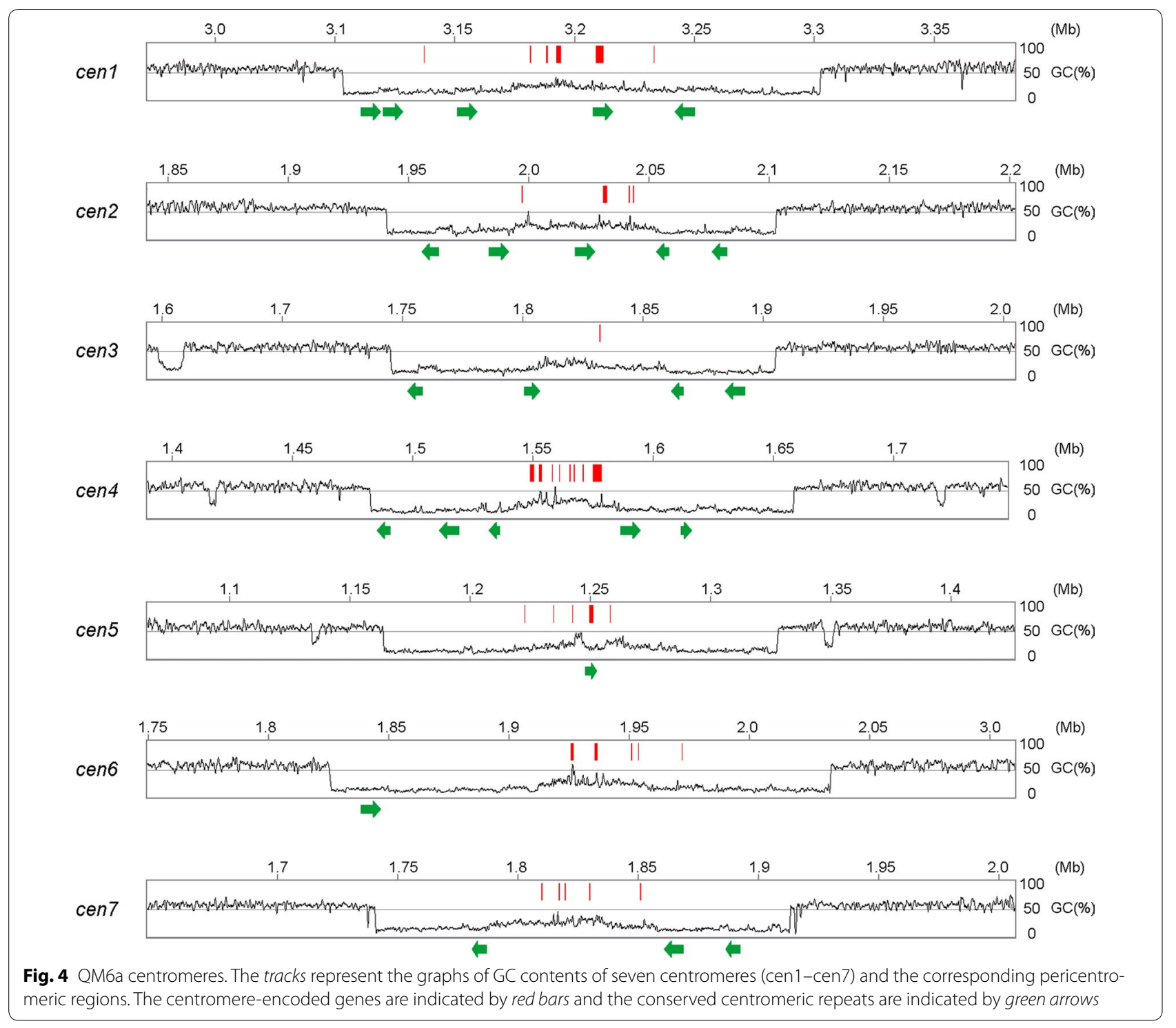

revealed that the seven QM6a centromeres collectively harbored 24 conserved sequences ( $\geq 90 \%$ identity; maximum length $8625 \mathrm{bp}$ and minimum length $4847 \mathrm{bp}$ ) with a copy number per chromosome ranging from one to five (Additional file 4). These conserved sequences are centromere-specific and highly AT-rich, perhaps representing centromeric repeats.

The seven QM6a centromeres also separately encode 13 previously annotated genes and 24 newly predicted genes (Additional file 3: Table C6). Centromere-encoded transcripts are known to be integral components of the genomes of mammals, higher plants, and the fission yeast Schizosaccharomyces pombe [31-34]. Copy numbers of these 37 centromere-encoded transcripts were relatively low; their expression (TPM $>0$ ) could only be detected in QM9414, but not in QM6a (Additional file 3: Table C6). The QM9414 transcriptomic data from the Illumina HiSeq 2000 platform [25] apparently had better sequencing depth than the QM6a transcriptomic data from the SOLiD platform [24]. It will be of importance to investigate whether these 24 centromeric repeats and 37 centromere-encoded genes are involved in centromere integrity and chromosome segregation fidelity in $T$. reesei.

The AT-rich chromosomal blocks next to the 14 telomeres are subtelomeres, with the shortest being $\sim 1 \mathrm{~kb}$ and the longest up to $87 \mathrm{~kb}$ (Additional file 1: Table A3). Other than centromeres and subtelomeres, there are 2328 interspersed AT-rich blocks (Table 2). On average, there are only five genes between two neighboring 
interspersed AT-rich blocks. The biological relevance of these interspersed AT-rich blocks remains to be elucidated (see below). It is worth noting that both the $5^{\prime}$ and $3^{\prime}$ flanking sequences of the rDNA locus contain a $2 \mathrm{~kb}$ AT-rich block (Fig. 3). We postulate that these two ATrich blocks might be involved in regulating nucleolar organization, rRNA transcription, rDNA copy homeostasis, and prevention of repeat-induced point mutation (RIP) (see below).

\section{Comparative genomic analyses of chromosome architectures}

Next, we compared the QM6a genome with 11 publicly available and well-assembled fungal genomes for their AT-rich blocks. We highlight that all these fungal genomes contain $\leq 0.11 \%$ unresolved or unknown bases (Ns) (Additional file 1: Table A12). Unresolved bases jeopardize the integrity of in silico genomic analysis.

All of these 12 fungal genomes have many short ATrich blocks with lengths of $0.5-3 \mathrm{~kb}$ (Table 2). In S. cerevisiae, these short AT-rich blocks correlate with the convergent intergenic regions and the pericentromeres that associate with cohesin (an evolutionarily conserved protein complex that functions to hold a pair of sister chromatids during mitosis and meiosis). The average distance between neighboring cohesin-binding sites along yeast chromosome arms is $10-15 \mathrm{~kb}$, which is compatible with the observed localized oscillations in base composition [35-37]. It has been suggested that sister chromatid connections via cohesin complexes occur preferentially at the chromosome axes, the bases of intrachromosomal loops, or topologically associated domains (TADs) [35, 38-40]. Expression of genes within TADs is somewhat correlated [41-45]. Intriguingly, the average distance between the neighboring AT-rich blocks along QM6a chromosomal arms is $\sim 13 \mathrm{~kb}$. We suggest that these ATrich blocks might be functionally associated with chromosomal loading of cohesin in $T$. reesei.

We were able to categorize these 12 fungal genomes into three different groups according to (1) the average AT content, (2) the genome-wide distribution of local AT content, and (3) the number of long ( $\geq 3 \mathrm{~kb}$ ) AT-rich blocks. For example, in the QM6a genome, there were 167 AT-rich blocks of length $\geq 3 \mathrm{~kb}$ (Table 2; Fig. 6).

The Group I fungi consist of six filamentous ascomycetes (Pezizomycotina), including QM6a, N. crassa (OR74A), Penicillium chrysogenum (P2niaD18), Mycosphaerella graminicola (IPO323), Fusarium fujikuroi (IMI 58289), and Aspergillus nidulans (FGSC A4). Their genomes not only have similar average AT contents $(\sim 50 \%)$ but also display biphasic local AT content distributions due to the presence of many longer ( $\geq 3 \mathrm{~kb})$ ATrich blocks (Table 2; Fig. 6a, top two panels). In N. crassa,
AT-rich blocks are DIM-3 (importin $\alpha$ )-dependent constitutive heterochromatins with transposon relicts and trimethylated histone 3 at lysine 9 (H3K9me3). Genome organization in $N$. crassa nuclei is largely defined by constitutive heterochromatins via strong intra- and interchromosomal contacts [46, 47].

Group II are three basidiomycetes, i.e., Ustilago maydis (521), Coprinopsis cinerea (Okayama 7\#130), and Cryptococcus neoformans (JEC21). Their average AT contents are $\sim 50 \%$, similar to those of Group I fungal genomes (Table 2). The Group II fungal genomes display relatively normal local AT content distribution (Fig. 5a, the lowest panel) due to the absence of longer AT-rich blocks (Fig. 5b).

Group III are three hemiascomycete yeasts, $S$. cerevisiae (S288C), S. pombe (972h-), and Candida glabrata (CBS138). Their average AT contents (62-64\%) are $12-14 \%$ higher than those of Group I and Group II fungi (Table 2). These three yeast genomes also display a relatively normal distribution of AT local content (Fig. 5a, the second lowest panel) and have no or very few longer AT-rich blocks (Fig. 5b).

\section{Repeat-induced point mutation (RIP) is identified in Trichoderma reesei}

The high number of long AT-rich blocks in the Group I fungal genomes might be correlated with RIP: a phenomenon originally discovered in $N$. crassa at a premeiotic stage during sexual development [46]. The process of RIP requires a specialized cytosine methyltransferase gene rid-1 (RIP-defective) and induces cytosine-to-thymine (C-to-T) point mutations in a homology-dependent manner $[48,49]$. Several previous studies found no evidence of RIP in any of the Group II or Group III fungi we investigated here [50-52]. RIP has been documented in $P$. chrysogenum [53], F. fujikuroi [54], M. graminicola [55], and $A$. nidulans [56], but it has never been experimentally demonstrated in any Trichoderma species $[18,57]$. Intriguingly, the QM6a complete genome encodes almost all proteins known to be involved in RIP and DNA methylation in $N$. crassa, including rid1 (TrC1298W/37515) [57], $\operatorname{dim} 2$ (TrB0908W/34985; DNA methyltransferase), $\operatorname{dim} 3$ (TrE0517W/73508; importin $\alpha$ ), $\operatorname{dim} 5$ (TrB0159C/111216; H3K9 methyltransferase) [13], dim7 (TrD0784W/74397), $\operatorname{dim} 8$ (TrG0915W/56582), $\operatorname{dim} 9$ (TrC0267C/108263), and hpo (TrB1406W/109088; HP1) (Additional file 2: Tables B1, B2).

We carried out both bioinformatic and molecular genetic analyses to determine whether RIP could operate in $T$. reesei. The RIPCAL software tool was applied to compare differences in the extent of RIP mutations of different sequences by determining two widely used RIP indices: TpA/ApT and [(CpA+TpG)/(ApC+GpT)] 


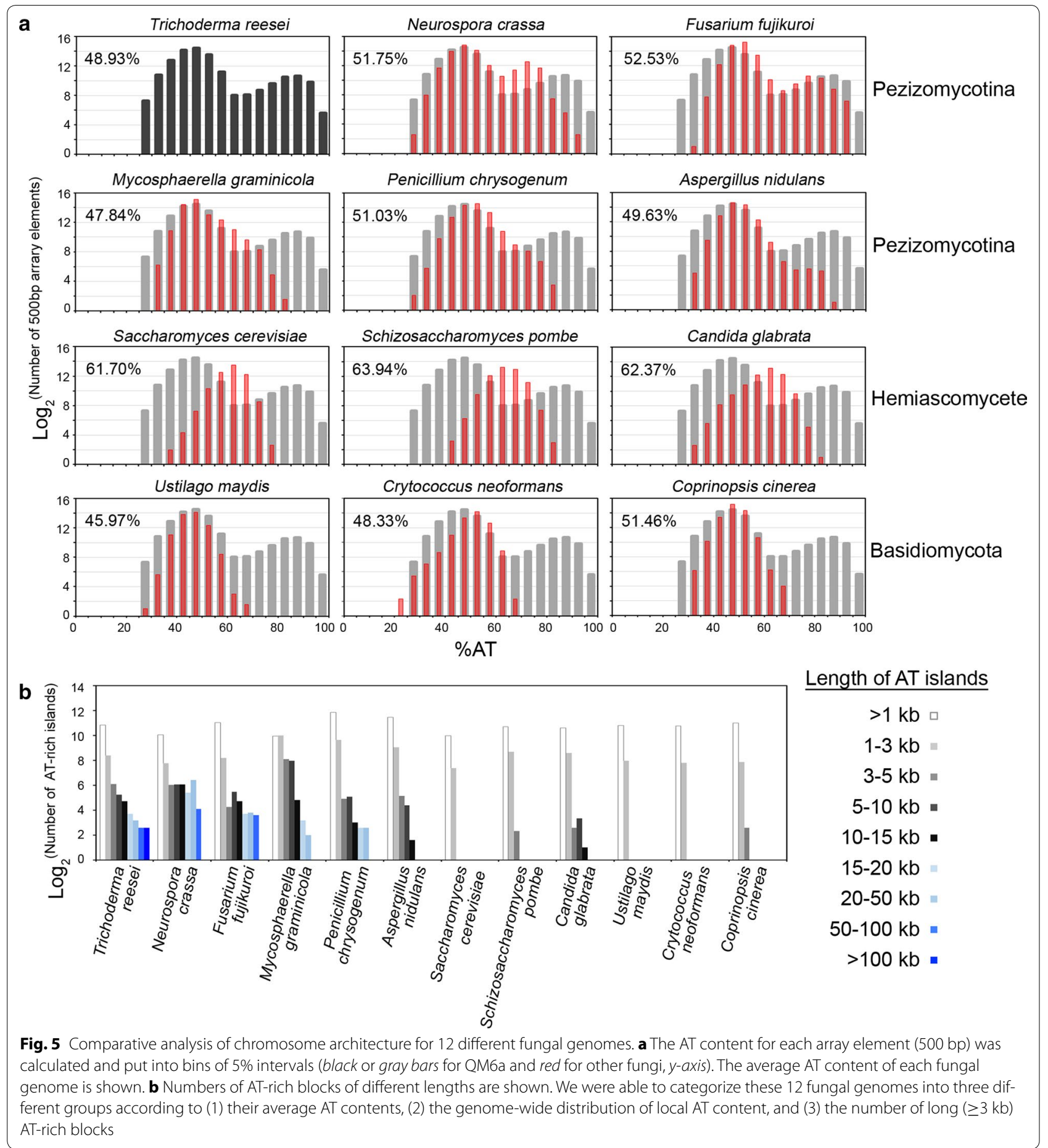

[58, 59]. Higher values of $\mathrm{TpA} / \mathrm{ApT}$ and lower values of $[(\mathrm{CpA}+\mathrm{TpG}) /(\mathrm{ApC}+\mathrm{GpT})]$ indicate stronger RIP responses $[60,61]$. We found that the hierarchy for RIP in QM6a is the mating-type gene MAT1-2-1 < all predicted genes $<$ the whole QM6a genome $<$ the large rDNA tandem repeats $(18 \mathrm{~S}-5.8 \mathrm{~S}-26 \mathrm{~S})<5 \mathrm{~S}$ rDNAs. It has been reported that the large rDNA tandem repeats and the 5S rDNAs in $N$. crassa survived RIPs due to either nucleolar sequestration [62] or their smaller size [60], respectively. The RIP indices of the mating gene (MAT1-2-1 or MFa), all predicted genes, and the whole genome suggest that the RIP responses in QM6a are 
a
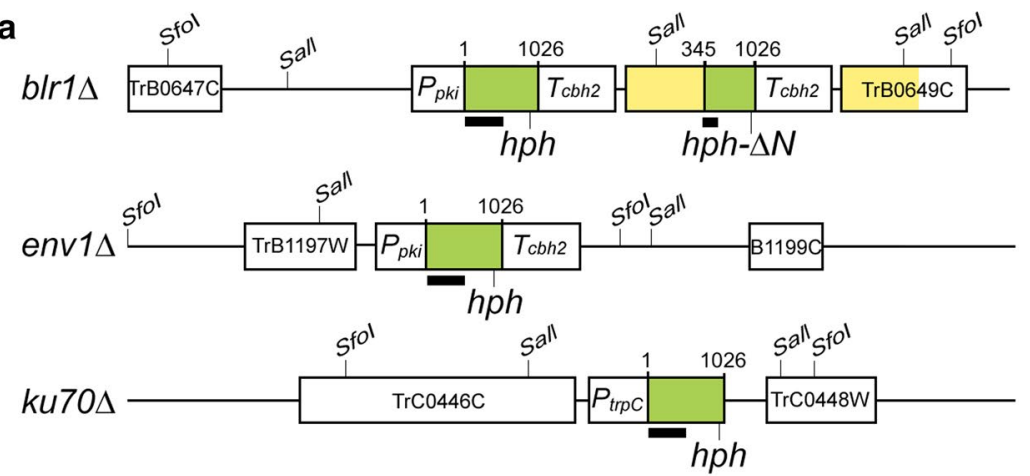

b

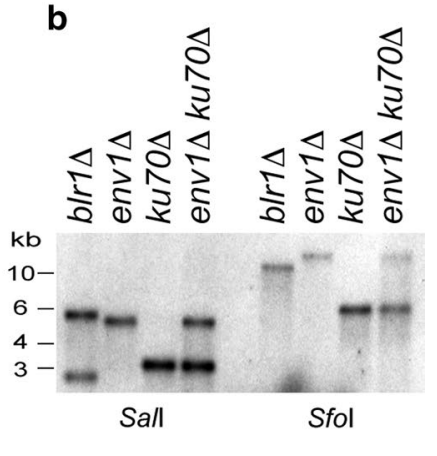

C

\begin{tabular}{|c|c|c|c|c|c|}
\hline \multicolumn{2}{|c|}{ Parental } & \multicolumn{4}{|c|}{ F1 progeny } \\
\hline MAT1-1 & MAT1-2 & 1 & 2 & 3 & 4 \\
\hline blr1 $\Delta \square$ & blr1 $\Delta$ & 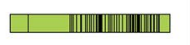 & 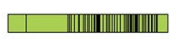 & 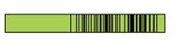 & 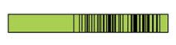 \\
\hline$b / r 1 \Delta \square$ & blr1 & & & inviable & 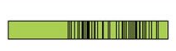 \\
\hline env1 $\Delta_{\square}$ & blr1s & 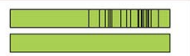 & 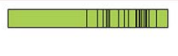 & & \\
\hline env1 $1 \Delta$ & env1 $\Delta \square$ & $\square$ & $\longrightarrow$ & $\square$ & $\square$ \\
\hline env1 $\triangle$ & ku70 $\triangle$ & & & & \\
\hline$k u 70 \Delta \square$ & ku70 & & & $\square$ & $\square$ \\
\hline$k u 70 \Delta$ & $k u 70 \Delta$ & $\square$ & $\square$ & & \\
\hline $\begin{array}{l}\text { ku70 } \\
\text { env1 } \square\end{array}$ & $\begin{array}{l}\text { ku70 } \\
\text { env1 }\end{array}$ & & | & 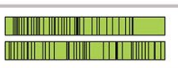 & 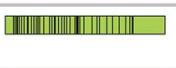 \\
\hline
\end{tabular}

Fig. 6 RIP in Trichoderma reesei. a Schematic diagram of the gene deletion cassettes in the three mutants. Each cassette consists of three components: the dominant hygromycin B-resistant marker open reading frame (hph; green box), flanked by an upstream promoter $\left(P_{\text {pki }}\right.$ or $\left.P_{\text {trpc }}\right)$, and/ or a downstream terminator $\left(T_{c b h 2}\right)$. The full-length hph gene has 1026 bps. A truncated $h p h-\Delta N_{304-1026}{ }^{-T}$ cbh2 cassette was spontaneously generated in blr1 $\triangle$ during transformation. The dark line underneath hph represents the DNA probe used for Southern hybridization. The upstream and downstream neighboring genes of the deleted gene are indicated by white boxes and their protein identity numbers. In blr $1 \Delta$, there are two identical 12960 fragments (in yellow) and two copies of $\mathrm{T}_{\mathrm{cbh2}}$ (in white). b Southern hybridization. Genomic DNA of indicated strains was digested by Sall or Sfo1, and then subjected to Southern blot analysis using the hph DNA probe shown in a. c Occurrence of RIP in the F1 progeny. The C-to-T point mutations in the respective full-length hph cassettes (in green) are indicted by vertical black bars. Only the results of one representative sexual crossing experiment $(n=10)$ are shown. The full-length hph cassette in each progeny was amplified by polymerase chain reaction (PCR) and then sequenced by Sanger's method

as robust as those in $N$. crassa (Additional file 1: Table A13). Thus, we suggest that $T$. reesei has evolved an RIP system similar to that of N. crassa. Our results also reveal that the longer the AT-rich blocks, the greater the extent of RIP mutations in QM6a (Additional file 1: Table A13), consistent with RIP mutations accumulating in AT-rich sequences.

Next, we carried out sexual crossing tests between four parental (F0) strains, including wild-type CBS999.97 [7], blr1 1 , env1 $[9,10]$, and ku70 $\Delta[63]$, and then tested their F1 progeny for mutations in the full-length hygromycin-resistant $(h p h)$ genes present in the selection marker construct used for deletion of the corresponding T. reesei genes [10]. All progeny were isolated using the hexadecad dissection technique [8]. No $h p h$ sequence is present in the wild-type CBS999.97 F0 strains, whereas env1 $1 \Delta$ and $k u 70 \Delta$ each carries one copy of full-length $h p h$. In contrast, the blr1s mutant contains two tandem head-to-tail hph sequences resulting in repeats: one is a full-length $\mathrm{hph}$ and the other an $\mathrm{N}$-terminal truncated $h p h-\Delta N$ (Fig. 6a). All of the hph and $h p h-\Delta N$ alleles in the corresponding parental strains were confirmed first by genomic PCR and Sanger sequencing (see Additional file 5 for their nucleotide sequences) and then by Southern hybridization (Fig. 6b).

After sexual crossing $(n=10)$, the $\mathrm{F} 1$ progeny displayed numerous $\mathrm{C}$-to- $\mathrm{T}$ point mutations in all cases where two similar sequences were present in one mating partner before crossing, i.e., in progeny of blr1 $\Delta$

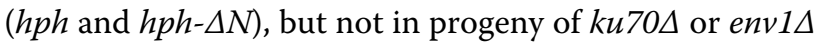


that comprise only one copy of full-length hph in their genome (Fig. 6c; Additional file 1: Table A14).

To determine whether RIP could operate between two genetically unlinked hph alleles, we generated a

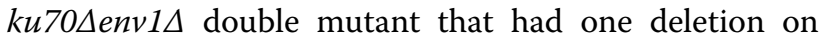

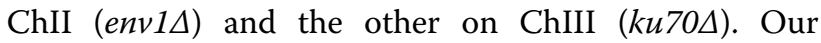
data revealed that sexual crossing $(n=10)$ between $k u 70 \Delta e n v 1 \Delta$ and a wild-type mating partner also resulted in C-to-T point mutations in progeny of these crosses (Fig. 6c; Additional file 5).

In N. crassa, sequences mutated by RIP showed skewed dinucleotide frequencies because of the sequence preference of RIP $(\mathrm{CpA}>\mathrm{CpT}>\mathrm{CpG}>\mathrm{CpC})$ [64]. By comparing the nucleotide sequences of the hph alleles in all $\mathrm{F} 1$ progeny (Additional file 5: Appendix A4), we found that T. reesei displayed a different sequence preference of RIP, i.e., $\mathrm{CpA} \approx \mathrm{CpG} \gg \mathrm{CpT}>\mathrm{CpC}$ (Additional file 1: Table A14).

We conclude that $T$. reesei, like $N$. crassa, exhibits high homology pairing and RIP activities at a premeiotic stage before premeiotic DNA synthesis. Our results are thus of tremendous importance for industrial strain improvement.

\section{Repetitive features}

The completeness of the QM6a and other 11 high-quality fungal genomes also allowed us to accurately survey genome-wide repetitive features and their correlation to RIP using the RepeatMasker search program (http:// www.repeatmasker.org/). We were able to identify almost all Ty elements along the 16 chromosomes of $S$. cerevisiae [65, 66] (Additional file 1: Table A15). Our results also confirm that the genome of $N$. crassa accumulates fragmented and rearranged transposon relics, in particular gypsy-LTRs (long terminal repeats) and Tad-LINEs (long interspersed repeat elements) [30]. The fungal wheat pathogen $M$. graminicola has a high copy number of gypsy-LTRs, copia-LTRs, and Tad-LINEs [55]. gypsyLTRs are highly overrepresented in the genomes of $S$. pombe [67], C. neoformans [68], and C. cinerea [69]. The Candida glabrata CBS138 genome contains very few $(\sim 0.4 \%)$ repetitive sequences (http://www.candidagenome.org/) (Additional file 1: Table A16).

Intriguingly, the QM6a genome has the fewest transposon sequences among the six Group I fungal genomes (Additional file 1: Table A16). The majority of copiaLTRs (6/8), gypsy-LTRs (9/10), CMC-EnSpm (6/6), and MULE-MuDR (13/21) are located in longer AT-rich blocks. In contrast, most LINEs (16/18) are located in non-AT-rich regions (Additional file 1: Table A17). Neither centromeres nor telomeres show a preponderance of any particular type of transposable element (Additional file 1: Table A17). According to their RIP indices (Additional file 1: Table A13) and smaller size (Additional file 1:
Tables A17, A18), we conclude that almost all transposon sequences in QM6a are fragmented or rearranged transposon relics.

The copy numbers of transposon sequences in QM6a we report here (Additional file 1: Table A16) are much lower than those reported by Kubicek et al. [13] using the QM6a-v2.0 draft. Both we and Kubicek et al. used the RepeatMasker search program to search for repetitive sequences. It is important to point out that the limitation of this widely used program is that it often generates many false-positive results. This is why we first applied it to the S. cerevisiae genome to determine the optimum parameters for filtering the preliminary RepeatMasker data (see "Methods"). The number and locations of five different Ty elements in all of that 16 yeast's chromosomes had been determined before $[65,66]$.

\section{Partitioning of gene clusters by AT-rich blocks}

A hallmark of the QM6a-v2.0 draft genome is that a third of the 228 CAZyme genes are non-randomly distributed and form several CAZyme gene clusters. Several of the regions of high CAZyme gene density also contain genes encoding proteins involved in secondary metabolism. Accordingly, it has been proposed that gene clustering and/or coexpression might be evolutionarily advantageous for $T$. reesei in its competitive soil habitat or other natural environments [11, 70]. Using the QM6a-HiC draft genome as a reference, 20 CAZyme gene clusters and 42 SSCP gene clusters were identified in the QM6a$\mathrm{HiC}$ genome draft [18]. All these gene clusters consisted of only 3-6 CAZyme and/or SSCP genes but, surprisingly, gene clustering did not influence gene expression.

To gain a better insight, we re-examined all these 62 CAZyme and SSCP gene clusters in QM6a-HiC [18]. Due to sequencing and assembly errors, some gene clusters are overlapped or even duplicated. The original 62 gene clusters locate to 46 chromosomal blocks in the complete QM6a genome. The majority of these gene clusters also contain new QM6a genes we annotate in this study (Additional file 2: Table B4). One gene cluster even contains a counterfeit gene (QM6a-v2.0 gene number 71245) [18].

Intriguingly, except for 5 short gene clusters $(\leq 4$ contiguous genes), all the other 41 gene clusters in the complete QM6a genome are divided into smaller compartments by AT-rich blocks (Additional file 2: Table B4). The first example of this phenomenon is a nitrate assimilation gene cluster and an annotated CAZyme gene cluster [18] immediately adjacent to tel2R. These two gene clusters were divided by tel2R and five interspersed AT-rich blocks into five smaller compartments (Fig. 7a). The first (or rightmost) compartment consists of one hypothetical protein gene (TrB1976W/111729) and three 


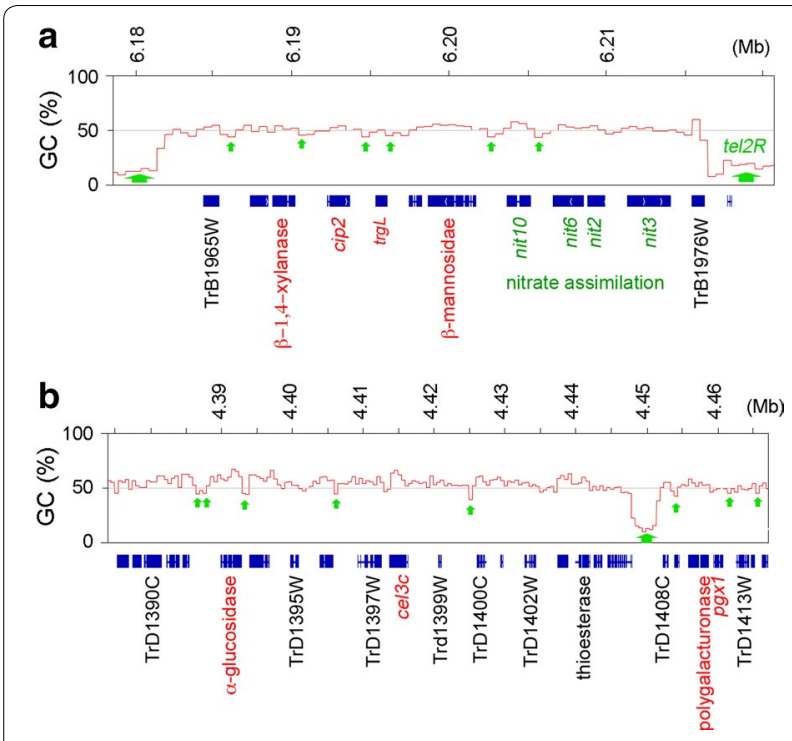

Fig. 7 Partitioning of gene clusters by AT-rich blocks. The tracks represent the graphs of GC contents (window size 500 bp) of a gene cluster next to tel2R (a) and pericentromeric regions $(\mathbf{b})$. All exons of all the predicted genes are indicated with blue squares, short AT-rich blocks by smaller green arrows, tel2R and long AT-rich blocks by larger green arrows, the CAZyme genes in red, the nitrate assimilation genes in dark green, and the systematic names of representative QM6a genes in black

nitrate reductase genes (nit3, $\operatorname{TrB} 1975 \mathrm{~W} / 81955$; nit2, TrB1974C/69210; nit6, TrB1973C/69291); transcripts of these genes were barely detectable (TPMs $<0.5)$ under glucose for $48 \mathrm{~h}$, then in straw for $24 \mathrm{~h}$, and finally with the addition of glucose for $5 \mathrm{~h}$. The second compartment comprises a sole nitrate transporter gene (nit10, TrB1972C/111724); nit10 was slightly induced by straw and then repressed by the addition of glucose. The third compartment contains a $\beta$-mannosidase gene, a member of glycoside hydrolase family 2 (GH2, TrB1971C/69245) and a hypothetical gene (TrB1970W/69181). Compared to nit10, this $\beta$-mannosidase gene exhibited $\sim$ fivefold greater induction by straw. The fourth compartment has only a $\beta$-1,4-glucuronan lyase gene (trgL, TrB1969W/69189). Like the nitrate reductase genes, $\operatorname{trgL}$ did not respond to glucose or straw substrate. Finally, the last (or leftmost) compartment harbors two CAZyme genes and a hypothetical protein gene (TrB1966/111717). The two CAZyme genes are the glucuronoyl esterase cip2 (TrB1968W/123940) and a GH30 endo- $\beta$-1,4-xylanase (TrB1967C/69276) (Fig. 7a). These two genes were highly induced by straw and then repressed by the addition of glucose (Additional file 1: Table A19A).

The second example is a CAZyme gene cluster on ChIV with 22 genes. It is divided by eight AT-rich blocks into seven smaller compartments $(\mathrm{C} 1-\mathrm{C} 7)$. The four CAZyme genes are located in different compartments: a GH31 $\alpha$-glucosidase (TrD1393W/82235) in C1, cel3c (TrD1398C/82227) in C3, and a GH28 polygalacturonase (TrD1411C/70186) and $p g x 1$ (TrD1412C/112140) in C6 (Fig. 7b). These four CAZyme genes were differentially regulated by straw and the addition of glucose (Additional file 1: Table A19B).

The third example is the CAZyme gene cluster close to tel7R. Six AT-rich blocks partitioned this gene cluster into four smaller compartments (C1-C4). The four CAZyme genes were allocated to three different compartments: the egl $2 /$ cel5 endo- $\beta-1,4$-glucanase $(\mathrm{TrG} 1193 \mathrm{~W} / 104060)$ in C1, GH79 $\beta$-glucuronidase (TrG1202W/72568) and rhamnogalacturonyl hydrolase (TrG1204W/57179) in $\mathrm{C} 2$, and the cbh cellobiohydrolase ( $\operatorname{TrG1206C/72567)~in~C4.~Only~}$ $c b h$ was highly induced by straw in QM6a (Additional file 1 : Table A19C).

Together, these results might explain why it was previously reported that gene clustering did not influence gene expression [18]. We propose that these smaller compartments are structurally and functionally similar to intrachromosomal loops or TADs.

It should be noted that occurrence of intergenic ATrich blocks did not always result in differential gene expression. For example, a gene cluster with three contiguous CAZyme genes-the acetyl xylan esterase gene axe1 (TrE0669C/73632; acetyl xylan esterase), the cellulose-induced protein cip1 (TrE0670C/73638), and the $\beta-1,6-N$-acetylglucosaminyl transferase gene egl4 (TrE0671C/73643)-resides in the middle of ChV. These three CAZyme genes are separated by four ATrich blocks into three smaller compartments, but they were all highly induced by straw and then repressed by the addition of glucose (Additional file 1: Table A19D). In this case, each gene becomes a partitioned functional unit (see "Discussion"). Simultaneous expression of the axe1-cip1-egl4 triad might be independently controlled by other determinants, e.g., common transcription factors and/or similar chromosomal conformation.

\section{Discussion}

Trichoderma reesei QM6a and its derivatives have been widely used for nearly four decades to produce plant cell wall-degrading enzymes and heterologous recombinant proteins. In this study, we have obtained a high-quality complete genome sequence of QM6a. We readily uncovered many sequencing, assembly, and gap-closing errors in earlier draft genomes. The seven telomere-to-telomere QM6a chromosomes can be used as better scaffolds for comparative genomic analyses, not only with industrial strains but also other T. reesei wild isolates (e.g., CBS999.97) and other species in the same genus (e.g., $T$. 
atroviride and T. virens). Our results also revealed much new genomic information never provided by earlier draft genomes, including 7 centromeres, 14 telomeres, 2328 AT-rich blocks, 1630 newly predicted genes, 37 centromere-encoded genes, and 24 centromeric repeats. Therefore, our complete QM6a genome sequence provides a comprehensive roadmap for further studies of this economically important fungus, including industrial strain improvements and elucidation of the functional relationships between sequences, gene products, and genome organization.

The central finding of this study is that $T$. reesei has evolved a robust RIP system. Firstly, the QM6a genome contains the lowest overall copy number of transposons among six studied filamentous ascomycetes (including $N$. crassa). Secondly, as in N. crassa, sexual crossing readily induced $\mathrm{C}$-to- $\mathrm{T}$ point mutations on both tandem and unlinked duplicated sequences in $T$. reesei. Thirdly, almost all 2349 AT-rich blocks in QM6a were predicted by the RIPCAL software program to be affected by RIP. Considerable evidence suggests that AT-rich blocks establish a link between DNA sequence and chromatin architecture. In N. crassa, AT-rich blocks form constitutive heterochromatins and mediate intra- and inter-chromosomal contacts [46, 47]. In $S$. cerevisiae, AT-rich blocks constitute the chromosome axes or the bases of chromosomal loops or TADs [35, 38-40]. In mammalian interphase chromosomes, the spatial distribution of AT-rich blocks (e.g., lamina-associated domains, LADs) and GC-rich blocks (e.g., TADs or chromosomal loops) are evolutionarily conserved. LADs preferentially interact with other LADs, whereas TADs exhibit more localized chromosomal domains [71]. Intriguingly, our results reveal that the rDNA locus of QM6a is surrounded by two interspersed AT-rich blocks. It would be of interest to further investigate whether and how these two interspersed AT-rich blocks are involved in preventing rDNA from being affected by RIP as well as in regulating nucleolar organization, rRNA transcription, and rDNA copy homeostasis.

From the results of this study, we postulate that RIP does not function solely as a genome defense mechanism to diminish the potentially deleterious effects caused by the spread of transposable elements. It may also have important roles in reshaping the $T$. reesei genome. We demonstrate that the widespread interspersed AT-rich blocks lead to genome-wide partitioning of the gene clusters in QM6a. Our findings can readily account for why gene clustering does not affect gene expression in T. reesei. Mechanistically, RIP-mediated C-to-T mutations presumably can transform duplicated sequences in a CAZyme gene cluster into interspersed AT-rich blocks, thus dividing an ancestral gene cluster with multiple
CAZyme or SSCP genes into multiple smaller compartments or TADs. Intriguingly, it has been reported previously that many pathogenic fungi (Leptosphaeria maculans, Magnaporthe oryzae, Fusarium spp.) comprise AT-rich blocks with RIP-affected effector genes and transposable elements $[72,73]$ and that RIP is a potential factor in L. maculans in creating the rapid sequence diversification (i.e., of the effector genes) needed for selection pressure [74] and concerted epigenetic regulation of their expression [75]. Partitioning of gene clusters by AT-rich blocks may also help to control simultaneous expression of the rDNA locus (Fig. 3) and of the three partitioned functional units in the axe1-cip1-egl4 triad (Additional file 1: Table A19D). Further research will reveal how RIP provides evolutionary advantages to $T$. reesei and other filamentous ascomycetes (Pezizomycotina) to survive in natural environments and pathogenic conditions.

\section{Conclusion}

The earlier genome drafts of QM6a and other T. ree$s e i$ industrial strains have been useful in identifying key genes involved in several important biological processes. However, due to numerous sequencing and assembly errors, they are not suitable for several other studies, e.g., genome-wide determination of gene order, chromosome architecture, and expression dynamics as well as chromosome engineering for genetic modification(s). The complete QM6a genome sequence provides an unprecedented opportunity to overcome those obstacles associated with earlier draft genomes. To avoid the limitations of working with incomplete datasets and the false leads that can come from trying to work with imperfect data, more caution should be exercised in utilizing the draft genome sequences solely determined by SGS and/or HiC for functional and comparative analyses.

\section{Methods \\ Fungal growth, DNA preparation, and pulsed-field gel electrophoresis (PFGE)}

QM6a was inoculated on petri-plates with malt extract agar (MEA) medium at $25^{\circ} \mathrm{C}$ until full asexual sporulation was observed ( $\sim 5$ days). $2 \times 10^{8}$ conidial spores were collected, and then inoculated in $50 \mathrm{~mL}$ potato dextrose medium (PDB) at $30^{\circ} \mathrm{C}$ for $6 \mathrm{~h}$. The germinated hyphae were harvested by centrifugation at $3000 \mathrm{~g}$ for $5 \mathrm{~min}$ at room temperature and incubated in $2 \mathrm{~mL}$ lysing enzyme buffer [0.1 $\mathrm{M} \mathrm{KH}_{2} \mathrm{PO}_{4}(\mathrm{pH} 5), 1.2 \mathrm{M}$ Sorbitol, $5 \%$ lysing enzyme (Sigma, USA)] at $30{ }^{\circ} \mathrm{C}$ for $1.5 \mathrm{~h}$. The protoplasts were harvested by centrifugation at $600 \mathrm{~g}$ for $10 \mathrm{~min}$ at $4{ }^{\circ} \mathrm{C}$, dissolved in $1.2 \mathrm{~mL} \mathrm{GuHCl}$ solution $(43 \%$ guanidine- $\mathrm{HCl}, 0.1 \mathrm{M}$ EDTA $\mathrm{pH}$ 8.0, $0.15 \mathrm{M} \mathrm{NaCl}, 0.05 \%$ Sarkosyl) at $65{ }^{\circ} \mathrm{C}$ for $20 \mathrm{~min}$ and then mixed with $6.4 \mathrm{~mL}$ 
ice-cold ethanol to precipitate the genomic DNA. The pellet was dissolved in $10 \times$ TE with $0.6 \mathrm{mg} / \mathrm{mL}$ RNase $\mathrm{H}$ at $37^{\circ} \mathrm{C}$ for $1 \mathrm{~h}$ and then in $0.4 \mathrm{mg} / \mathrm{mL}$ proteinase $\mathrm{K}$ at $65{ }^{\circ} \mathrm{C}$ for $1 \mathrm{~h}$. The genomic DNA was purified with the phenol:chloroform:isoamyl alcohol (25:24:1) method and then recovered by standard precipitation with ethanol. Next, the quality of high molecular weight genomic DNA for Illumina MiSeq and PacBio sequencing was validated by PFGE. The genomic DNA was separated in a $1 \%$ agarose gel in $0.5 \times$ TBE buffer, using a CHEF DR II (Biorad) with $0.5 \times$ TBE running buffer, continuously refrigerated at $14{ }^{\circ} \mathrm{C}$ and $6 \mathrm{~V} / \mathrm{cm}$ (current $110-125 \mathrm{~mA}$ ) for $18 \mathrm{~h}$. The Lambda DNA-Mono Cut Mix (New England Biolabs, N3019S) was used as size marker. Visualization was performed after staining with ethidium bromide after the electrophoresis.

\section{DNA sequencing and de novo assembly}

Illumina MiSeq sequencing was carried out at the DNA sequencing facility in the Institute of Molecular Biology, Academia Sinica (Taipei, Taiwan) in May 2016 (sequencing was coordinated by the author SYT). A shotgun paired-end library (average size of $550 \mathrm{bp}$ ) was prepared using the Illumina TruSeq DNA nano Sample Prep Kit. The Illumina library (including PCR amplification and quantification) was prepared automatically by the NeoPrep Library Prep System, and then sequenced on the MiSeq platform (300 cycles, paired-end sequencing) with the MiSeq Control software version 2.5.1 and Sequencing Analysis Viewer version 1.8.20. Sequencing data were sent to Illumina BaseSpace automatic analysis during running. For report version 2.2.9 with MiSeq 600 cycler V3 chemistry, $96.16 \%$ of clusters passed the filter and $78.32 \%$ of bases qualified higher than Q30 at $2 \times 300 \mathrm{n}$.

For PacBio continuous long-read sequencing, highquality genomic DNA was submitted to the Ramaciotti Centre for Genomics (University of New South Wales, Sydney, Australia). Sequencing was coordinated by Carolina Correa and Tonia Russel. High molecular weight DNA was sheared with g-TUBE (Covaris PN 520079), aiming at DNA fragments of about $20 \mathrm{~kb}$. The library was constructed with a $20 \mathrm{~kb}$ size-selected protocol using DNA Template Prep Kit 2.0 (PN 001-540-726), purified, and further selected for long insert size with a $0.35 \times$ AMPure (AMPure PB PN 100-265-900) bead size selection. The library was sequenced on a PacBio RSII device using the reagents DNA/Polymerase Binding Kit P4 (PN 100-236-500), DNA Sequencing Reagent 2.0 PN (100216-400), SMRT ${ }^{\circledR}$ Cell V3 (PN 100-171-800), and MagBead (PN 100-133-600), loading at 200 pM on the plate. Data were collected with Stage Start and 180 min movies. Seven SMRT ${ }^{\circledR}$ Cell cells were used to generate 263,312 reads and 3,397,762,180 bases.
The PacBio data were assembled with the SMRT Analysis Software v2.3.0 (http://www.pacb.com/productsand-services/analytical-software/smrt-analysis/). The assembly was performed with HGAP (3.0 protocol) with the following parameters: (1) PreAssembler Filter v1 (minimum sub-read length $=500 \mathrm{bp}$, minimum polymerase read quality $=0.80$, minimum polymerase read length $=100 \mathrm{bp}$ ); (2) PreAssembler v2 (minimum seed length $=10,000 \mathrm{bp}$, number of seed read chunks $=6$, alignment candidates per chunk $=10$, total alignment candidates $=24$, min coverage for correction $=6$ ); (3) AssembleUnitig v1 (Genome Size 34,000,000 bp, target genome coverage $=30$, overlap error rate $=0.06$, minimum overlap $=100 \mathrm{bp}$ and overlap $\mathrm{k}$-mer $=22$ ); (4) BLASR v1 mapping of reads for genome polishing with Quiver ( $\max$ divergence percentage $=30$, minimum anchor size $=12$ ). The final assembly contained nine unitigs (seven telomere-to-telomere chromosomes, a circular mitochondrial genome, and a $1870 \mathrm{bp}$ short sequence) with a total $34,993,035$ bp and approximately $87 x$ genome coverage. N50 was 5,311,312 bp and max was $6,835,650 \mathrm{bp}$. The per-base quality value $(\mathrm{QV})$ is higher than 45 and a little less than 50 (99.999\% accuracy; average 48.8). A BLASTN search revealed that the circular mitochondrial genome $(42,130 \mathrm{bp})$ was completely identical in nucleotide sequence with the QM9414 mitochondrial genome (Accession: NC_003388.1) [21]. The shortest unitig (1870 bp) is likely contaminating DNA because no Illumina MiSeq reads were mapped to its sequence.

\section{Mapping of Illumina reads over three different genomic drafts}

The two runs of Illumina MiSeq with 300-bp pairedend reads were combined into one. The forward and reverse data were $6.8 \mathrm{~Gb}$. Reads were preprocessed with Trimmomatic V0.32 (http://www.usadellab.org/ $\mathrm{cms} /$ ?page $=$ trimmomatic) to trim and remove reads $(33.15 \%)$ that fell below a quality score threshold of 30 (Q30) and were shorter than $30 \mathrm{bp}$. Next, CLC Genomics Workbench 7.5 (http://www.clcbio.com/blog/clc-genomics-workbench-7-5/) was used to map the qualified reads to three different QM6a genomic drafts: (1) QM6a-v2.0 (http://genome.jgi.doe.gov/Trire2/Trire2.home.html) plus the complete mitochondrial genome [20]; (2) the HiC draft genome [16] plus the complete mitochondrial genome [20]; and (3) the PacBio de novo assembly (this study). The parameters used for mapping were (1) mismatch $\operatorname{cost}=3$; (2) insertion cost $=2$; (3) deletion cost $=2$; (4) length fraction $=0.8$; and (5) similarity fraction $=0.8$. The results are listed in Additional file 1: Table S4. There were some differences between the PacBio and Illumina platforms, so the mapping results were used to extract a consensus sequence to adjust the bases to a final version 
of the chromosome sequences. We defined a threshold $=2$ to identify low coverage regions. For low coverage regions (i.e., threshold $\geq 2$ ), sequences from the Illumina MiSeq platform were used to construct the consensus sequence.

The seven telomere-to-telomere chromosomes were categorized with Roman numerals (ChI-ChVII), from the largest to the smallest. Artemis was used to determine the percentage of $\mathrm{G}+\mathrm{C}$ in $500 \mathrm{bp}$ non-overlapping windows. The centromere of each chromosome was their longest interspersed AT-rich block. The EMBOSS revseq software tool (v6.5.7; http://www.bioinformatics.nl/cgi-bin/emboss/revseq) was used to reverse and complement the nucleotide sequences of ChII, ChIV, and ChVI, respectively, so that all seven chromosomes had a shorter (left) arm at $5^{\prime}$ termini and a longer (right) arm at $3^{\prime}$ termini. The final sequences were first compared to the original version using BLAST searches (Additional file 1: Table A4) before being submitted to NCBI (Accession Numbers CP016232-CP016238).

\section{Genome reannotation}

The MAKER v2.31.8 (http://www.yandell-lab.org/software/ maker.html) genome annotation pipeline [74] was applied for genome reannotation. Firstly, all (28748) T. reesei protein sequences from NCBI were used for ab initio gene predictions. Secondly, the Augustus v3.0.3 gene prediction software (http://augustus.gobics.de/) [75] was also used to predict new genes. All proteins from Neurospora spp. and Fusarium spp. were used for Augustus training to impose constraints on the predicted gene structure, including splice sites, translation initiation sites, and stop codons. Thirdly, we isolated QM6a poly(A) RNA and performed a MiSeq RNA sequencing experiment. The resulting mRNA reads (trimming quality $=30$, min length $=20 \mathrm{bp}$ ) were applied to Trinity (v2.0.6; https:/github.com/trinityrnaseq/trinityrnaseq/wiki) for de novo transcriptome assembly. The expressed sequence tag (EST) results predicted by "Maker" were used to identify new genes. Finally, we integrated all the ESTs and protein sequences from the two publicly available databases (QM6av2.0 and RUT-C30-v1.0; at JGI) as well as all the ESTs assembled from two published transcriptome datasets [24, 25].

\section{A. QM6a-v2.0}

(http://genome.jgi.doe.gov/Trire2/Trire2.home.html)

TreeseiV2_FilteredModelsv2.0.transcripts.fasta

TreeseiV2_FilteredModelsv2.0.proteins.fasta

\section{B. RUT-C30-v1.0}

(http://genome.jgi.doe.gov/TrireRUTC30_1/TrireRUTC30_1.home.html)

TrireRUTC30_1_GeneCatalog_transcripts_20110526. nt.fasta
TrireRUTC30_1_GeneCatalog_proteins_20110526. aa.fasta

\section{The Illumina HiSeq 2000 sequencing reads from QM9414 treated with cellulose $(24,48$, and $72 \mathrm{~h})$, sophorose $(2,4$, and $6 h$ ), and glucose (24 and $48 h$ ) [25]}

GSE53629 (http://www.ncbi.nlm.nih.gov/geo/query/ acc.cgi?acc $=$ GSE53629)

SRR1057947: QM9414 Cellulose replication 1 SRR1057948: QM9414 Cellulose replication 2 SRR1057949: QM9414 Cellulose replication 3 SRR1057950: QM9414 Sophorose replication 1 SRR1057951: QM9414 Sophorose replication 2 SRR1057952: QM9414 Sophorose replication 3 SRR1057953: QM9414 Glucose replication 1 SRR1057954: QM9414 Glucose replication 2 SRR1057955: QM9414 Glucose replication 3

The Illumina-HiSeq.sra files were converted into fastq using the fastq-dump program of the SRA Toolkit. Trimmomatic v0.32 [76] was used to trim and remove reads that fell below a quality score threshold of 30 (Q30) and that were shorter than $10 \mathrm{bp}$. The Cufflinks pipeline tools (http://cole-trapnell-lab.github.io/cufflinks/) were downloaded for transcriptome assembly and differential expression analysis, including bowtie2-build (bowtie v2.2.3), tophat2 (v2.0.13), and cufflinks (v2.2.1). For read alignment, the parameters of tophat2 were as follows: (1) min-intron-length: 20 bp; (2) max-intron-length: $5000 \mathrm{bp}$ (to reflect introns and splicing elements of five diverse fungi); and (3) no transcript GTF (Gene Transfer Format) file was provided to guide assembly. There were $\sim 95 \%$ trimmed paired reads that could be aligned to the QM6a complete genome. After each QM9414 RNA-seq raw dataset was assembled into transcripts, the cuffmerge program was used to merge all GTF files into one. The gffread program was used to extract transcript sequences.

\section{$D$. The SOLiD sequencing reads from QM6a grown first in glucose for $48 \mathrm{~h}$, then in straw for $24 \mathrm{~h}$, and finally with the addition of glucose for $5 \mathrm{~h}$ [24]}

GSE44648 (http://www.ncbi.nlm.nih.gov/geo/query/acc. cgi?acc $=$ GSE44648 )

SRR764963: QM6a Glucose $48 \mathrm{~h}$ replication 1

SRR764964: QM6a Glucose 48 h replication 2

SRR764965: QM6a Glucose 48 h replication 3

SRR764966: QM6a Straw 24 h replication 1

SRR764967: QM6a Straw 24 h replication 2

SRR764968: QM6a Straw 24 h replication 3

SRR764969: QM6a Straw + Glucose 5 h replication 1

SRR764970: QM6a Straw + Glucose 5 h replication 2 
The SOLID.sra files were converted into csfasta and QV.qual using the abi-dump program of the SRA Toolkit. For transcriptome assembly and quantification, bowtiebuild (bowtie v1.1.0), tophat2, and cufflinks were used. All parameters were as for the QM9414 dataset, except the colorspace option was used for the bowtiel program. There were $\sim 33 \%$ reads that could be aligned to the QM6a complete genome.

Next, we performed gene filtering to finalize all predicted protein sequences, the filtering order was all $T$. reesei protein sequences from NCBI > Neurospora spp. and Fusarium spp. (Augustus v3.0.3) $>$ de novo assembly QM6a-RNA (Trinity v2.0.6) > QM6a v2.0 + RutC-30 v1.0 + QM6a-RNA (SOLiD) + QM9414-RNA (Illumina HiSeq 2000).

We also manually integrated almost all the annotation results reported by the Trichoderma research community $[4,18,24,25]$. We annotated 10786 predicted genes that covered almost all previously annotated genes in QM6av2.0 (9105 out of 9129) and RUT-C30-v1.0 (9717 out of 9852), respectively. Of these predicted QM6a genes, 904 were previously considered to be RUT-C30-specific. We successfully annotated several evolutionarily conserved DNA repair genes, including rad50, sae2/com1/CtIP, rad51, rad57, srs2, and pif1 (Additional file 2: Tables B1, B2). These genes were not annotated in QM6a-v2.0 (JGI), RUT-C30-v1.0 (JGI), and QM6a-HiC [18].

Finally, the BLASTP program was applied to compare our final predicted protein sequences to several publicly available protein databases, including NCBI nonredundant (nr) database, Universal Protein Resource (UniProt) Prot v2.0, N. crassa database (Broad Institute), F. fujikuroi (JGI), Sordaria macrospora (NCBI), $S$. cerevisiae (SGD), and S. pombe (PomBase and NCBI). The e-values of all BLAST results were $<1.0 \times 10^{-5}$. The Retrieve/ID mapping tool (UniProt) was used to map gene ID (UniProt sprot v2.0 and NCBI BLAST result) and to determine gene ontology (GO) (Additional file 2: Table B2).

Next, the bowtie-tophat program was used to align all QM6a (SOLiD) and QM9414 (Illumina MiSeq) RNA reads with the following parameters: (1) min-intronlength: $20 \mathrm{bp}$; (2) max-intron-length: $5000 \mathrm{bp}$ (because it was reported previously that the ranges of intron length in S. cerevisiae, A. nidulans, and N. crassa are 52-1002, 27-1903, and 46-1740 bp, respectively [76]); and (3) a transcript GTF (Gene Transfer Format) file was provided. To calculate read counts for each transcript, we applied featureCounts [77]. The values of TPM [26] were used as expression values: TPM = (individual gene RPK/ the sum of all RPKs) $\times 10^{6}$, whereas reads per kilobase $(\mathrm{RPK})=($ read counts/transcription length) (Additional file 2: Table B1, Additional file 3).

\section{RepeatModeler and RepeatMasker}

Novel repeat elements were identified by RepeatModeler-1.0.4 (http://www.repeatmasker.org/RepeatModeler. html) with default parameters. RepeatMasker (version 4.0.6) and the Repbase Library (http://www.repeatmasker.org) were used to scan 12 different fungal genomes for interspersed repeats and low complexity DNA sequences. The output of the program is a detailed annotation of the repeats that are present in the query sequence, as well as a modified version of the query sequence in which all the annotated repeats have been masked (default: replaced by Ns). To obtain high-confidence data, we first analyzed the genome sequences of $S$. cerevisiae because the number and location of five different Ty elements in all its 16 yeast chromosomes had been reported previously $[65,66]$. When the preliminary RepeatMasker data were filtered with two parameters (length $\geq 140$, SmithWaterman local similarity scores $\geq 450$ ), the final data (Additional file 1: Table A15) were quite consistent with the known results [65]. The same parameters were then applied to scan all other studied fungal genomes (Additional file 1: Table A16).

\section{RIP and RIPCAL}

The latest RIPCAL program (https://sourceforge.net/ projects/ripcal/) was downloaded [58, 59]. An initial analysis was done to predict if the complete QM6a genome sequence had been mutated by RIP. All strains used in this study had been previously described [10]. The methods for sexual crossing, single ascospore isolation, and preparation of genomic DNA have also been described before $[8,10]$. The full-length hph cassettes in all F0 parental strains and representative F1 progeny were amplified by PCR and analyzed by Sanger sequencing technology. All the nucleotide sequences of primers and the hph cassettes are listed in Additional file 1: Table A9 and Additional file 5, respectively. The RIPCAL dinucleotide frequency and EMBOSS compseq tool were used to determine the values of two RIP indices TpA/ApT and $[(\mathrm{CpA}+\mathrm{TpG}) /(\mathrm{ApC}+\mathrm{GpT})]$, respectively.

\section{Additional files}

Additional file 1: Table A1. Preliminary assembly results obtained by the Hierarchical Genome Assembly Process (HGAP 3.0). Table A2. Error corrections of the seven PacBio unitigs using the Illimina-MiSeq reads. Table A3. Characteristics and assembly of the seven QM6a chromosomes. Table A4. Mapping of all trimmed paired-end Illumina MiSeq reads to three QM6a genome drafts. Table A5. The MiSeq reads mapped to the QM6a-v2.0 draft genome. Table A6. Sixteen false predicted genes in QM6a-v2.0. Table A7. The QM6a-v2.0 draft genome compared to the complete QM6a genome sequence. Table A8. The HiC draft genome compared to the complete QM6a genome sequence. Table A9. PCR primers. Table A10. The complete QM6a genome sequence versus the 
RUT-C30-v1.0 draft genome. Table A11. Gene Ontology of some newlypredicted genes. Table A12. Sequencing quality of 18 different fungal genomes. Table A13. RIP indices of various sequences in QM6a and Neurospora crassa. Table A14. Repeat-induced C-to-T mutations observed in the hph alleles in all F1 progeny. Table A15. Saccharomyces cerevisiae Ty elements by chromosome. Table A16. Transposable elements in 12 well-assembled fungal genomes. Table A17. Repetitive sequences in different chromosomal regions. Table A18. Repetitive sequences in seven QM6a chromosomes. Table A19. Partitioning of four gene clusters by the AT-rich islands. The TPM values in glucose (48 hrs), in straw (24 hrs) and in straw (24hrs) then glucose (5 hrs) are shown [24].

Additional file 2: Table B1. Genome annotation of the complete QM6a genomes, including AT-rich blocks, predicted genes, repetitive features, gene ontology (GO), gene names in T. reesei, Saccharomycetes cerevisiae and Neurospora crassa, as well as gene identity numbers in QM6a-v2.0 and RUT-C30-v1.0. (Columns A-H) All the genes that were not annotated in QM6a-v.20 and Rut-C30-v1.0 are highlighted with yellow background, whereas those only annotated in Rut-C30-v1.0 are highlighted with yellow-green background. (Columns O-AE) Comparative transcriptomic analysis of all QM6a genes in different carbon sources using two published transcriptome datasets: (1) The SOLiD sequencing reads from QM9a grown first in glucose for 48 hours, then in straw for 24 hours and finally with the addition of glucose for 5 hours [24]; (2) The Illumina HiSeq 2000 sequencing reads from QM9414 treated with cellulose $(24,48$ and 72 hours), sophorose ( 2,4 and 6 hours) and glucose ( 24 and 48 hours) [25]. The TPM values are shown. Table B2. Annotation of the complete QM6a genome. All the annotated QM6a genes are compared to those in QM6a-v2.0, Rut-C30-v1.0, all Trichoderma reesei proteins from NCBI, the QM6a-HiC annotation results by Druzhinina et al. [18], the annotation results by Schmoll et al. [4], as well as the BLAST results from Uniprot, NCBI non-redundant (nr) database, Fusarium fujikuroi (JGI), Sordaria macrospora (NCBI), Saccharomyces cerevisiae (SGD) and Schizosaccharomyces pombe (PomBase and NCBI). The e-values of all BLASTP results were $<$ $1.0 \times 10^{-5}$. Table B3. TPM values of all new QM6a genes revealed by two publicly-available transcriptome datasets $[24,25]$. Table B4. Locations of 62 previously identified CAZyme and SSCP gene clusters [18] in 42 chromosomal blocks of the complete QM6a genome. All previously annotated QM6a-v2.0 or QM6a-HiC genes in these gene clusters are indicated in blue. AT-rich islands in these 42 chromosomal blocks are indicated in red. All new QM6a genes we identified in the complete QM6a genome are indicated in darkgreen.

Additional file 3: Table C1. Straw-induced QM6a-v2.0 genes identified by Ries et al. [24] and their TPM values. Table C2. Straw-induced QM6av2.0 genes not identified by Ries et al. [24] and their TPM values. Table C3. New QM6a genes that are highly induced ( $\geq 20$-fold) by straw and their TPM values. Table C4. New QM6a genes that are significantly induced (5-20-fold) by straw and their TPM values. Table C4. Two new QM6a genes that are highly induced by cellulose and their TPM values. Table C5. Nine new genes having higher TPM values in QM6a than in QM9414. Table C6. Centromere-encoded genes and their TPMs in QM9414.

Additional file 4. Sequence alignments of the 24 centromeric repeats.

Additional file 5. Nucleotide sequences of the $h$ gh alleles from all strains listed in Figure 7 .

\section{Authors' contributions}

WCL and CLC isolated the fungal genomic DNA for sequencing and conducted the sexual crossing experiments. CHH, WCL, and YCC carried out bioinformatic analyses. SYT performed Illumina MiSeq sequencing experiments. TFW conceived and designed the experiments, and analyzed the data. TFW and WCL wrote the paper. All authors read and approved the final manuscript.

\section{Author details}

${ }^{1}$ Taiwan International Graduate Program in Molecular and Cellular Biology, Academia Sinica, Taipei 115, Taiwan. ${ }^{2}$ Institute of Life Sciences, National Defense Medical Center, Taipei 115, Taiwan. ${ }^{3}$ Institute of Molecular Biology, Academia Sinica, Taipei 115, Taiwan. ${ }^{4}$ Institute of Genome Sciences, National Yang-Ming University, Taipei 112, Taiwan.

\section{Acknowledgements}

We thank Paul Hsu at the IMB Bioinformatics Service Core, Yu-Tang Huang at the IMB Computer Room and the IMB Genomics Core for technical support, and John O'Brien for English editing. TFW would like to thank Frédérique Bidard-Michelot (IFP Energies Nouvelles, Paris, France) for personal communications on unpublished results of RUT-C30 genome assembly and a common consensus for QM6a chromosome numbering, ordering, and orientation.

\section{Competing interests}

The authors declare that they have no competing interests.

\section{Availability of data and materials}

All data generated or analyzed during this study are included in this published article and its Additional files 1, 2, 3, 4, and 5. Data deposition: BioProject (PRJNA325840), BioSample (SAMN05250858), QM6a genomic DNA illumina MiSeq (SRR4417032), QM6a Poly(A) RNA illumina MiSeq (SRR5229930), and the nucleotide sequences of seven complete QM6a chromosomes (CP016232-CP016238).

\section{Ethical approval}

This study needed no ethical approval and had no experimental research on humans.

\section{Funding}

TFW received supports from Academia Sinica, Taipei, Taiwan. The funding body was not involved in the design of the study and collection, analysis, and interpretation of data and in writing the manuscript.

\section{Publisher's Note}

Springer Nature remains neutral with regard to jurisdictional claims in published maps and institutional affiliations.

Received: 15 November 2016 Accepted: 22 May 2017

Published online: 03 July 2017

\section{References}

1. Reese ET. History of the cellulase program at the U.S. army Natick Development Center. Biotechnol Bioeng Symp. 1976:9-20.

2. Peterson R, Nevalainen H. Trichoderma reesei RUT-C30-thirty years of strain improvement. Microbiology. 2012;158:58-68.

3. Glass NL, Schmoll M, Cate JH, Coradetti S. Plant cell wall deconstruction by ascomycete fungi. Annu Rev Microbiol. 2013;67:477-98.

4. Schmoll M, Dattenbock C, Carreras-Villasenor N, Mendoza-Mendoza A, Tisch D, Aleman MI, Baker SE, Brown C, Cervantes-Badillo MG, Cetz-Chel J, et al. The genomes of three uneven siblings: footprints of the lifestyles of three Trichoderma Species. Microbiol Mol Biol Rev. 2016;80:205-327.

5. Bischof RH, Ramoni J, Seiboth B. Cellulases and beyond: the first 70 years of the enzyme producer Trichoderma reesei. Microbial Cell Fact. 2016;15(1):106.

6. Druzhinina IS, Kubicek CP. Familiar stranger: ecological genomics of the model saprotroph and industrial enzyme producer Trichoderma reesei breaks the stereotypes. Adv Appl Microbiol. 2016;95:69-147.

7. Seidl V, Seibel C, Kubicek CP, Schmoll M. Sexual development in the industrial workhorse Trichoderma reesei. Proc Natl Acad Sci. 2009;106:13909-14

8. Chuang YC, Li WC, Chen CL, Hsu PW, Tung SY, Kuo HC, Schmoll M, Wang TF. Trichoderma reesei meiosis generates segmentally aneuploid progeny with higher xylanase-producing capability. Biotechnol Biofuels. 2015:8:30.

9. Seibel C, Tisch D, Kubicek CP, Schmoll M. ENVOY is a major determinant in regulation of sexual development in Hypocrea jecorina (Trichoderma reesei). Eukaryot Cell. 2012;11:885-95.

10. Chen CL, Kuo HC, Tung SY, Hsu PW, Wang CL, Seibel C, Schmoll M, Chen RS, Wang TF. Blue light acts as a double-edged sword in regulating sexual development of Hypocrea jecorina (Trichoderma reesei). PLoS ONE. 2012;7:e44969. 
11. Martinez D, Berka RM, Henrissat B, Saloheimo M, Arvas M, Baker SE, Chapman J, Chertkov O, Coutinho PM, Cullen D, et al. Genome sequencing and analysis of the biomass-degrading fungus Trichoderma reesei (syn Hypocrea jecorina). Nat Biotechnol. 2008;26:553-60.

12. Koike H, Aerts A, LaButti K, Grigoriev IV, Baker SE. Comparative genomics analysis of Trichoderma reesei strains. Ind Biotechnol. 2013;9:352-67.

13. Kubicek CP, Herrera-Estrella A, Seidl-Seiboth V, Martinez DA, Druzhinina IS, Thon M, Zeilinger S, Casas-Flores S, Horwitz BA, Mukherjee PK, et al. Comparative genome sequence analysis underscores mycoparasitism as the ancestral life style of Trichoderma. Genome Biol. 2011;12:R40.

14. Arvas M, Pakula T, Smit B, Rautio J, Koivistoinen H, Jouhten P, Lindfors E, Wiebe M, Penttila M, Saloheimo M. Correlation of gene expression and protein production rate-a system wide study. BMC Genom. 2011;12:616.

15. Hakkinen M, Valkonen MJ, Westerholm-Parvinen A, Aro N, Arvas M, Vitikainen M, Penttila M, Saloheimo M, Pakula TM. Screening of candidate regulators for cellulase and hemicellulase production in Trichoderma reesei and identification of a factor essential for cellulase production. Biotechnol Biofuels. 2014;7:14

16. Vitikainen M, Arvas M, Pakula T, Oja M, Penttila M, Saloheimo M. Array comparative genomic hybridization analysis of Trichoderma reesei strains with enhanced cellulase production properties. BMC Genom. 2010;11:441.

17. Marie-Nelly H, Marbouty M, Cournac A, Flot JF, Liti G, Parodi DP, Syan S, Guillen N, Margeot A, Zimmer C, et al. High-quality genome (re)assembly using chromosomal contact data. Nat Commun. 2014;5:5695.

18. Druzhinina IS, Kopchinskiy AG, Kubicek EM, Kubicek CP. A complete annotation of the chromosomes of the cellulase producer Trichoderma reesei provides insights in gene clusters, their expression and reveals genes required for fitness. Biotechnol Biofuels. 2016;9:75.

19. Kim KE, Peluso P, Babayan P, Yeadon PJ, Yu C, Fisher WW, Chin CS, Rapicavoli NA, Rank DR, Li J, et al. Long-read, whole-genome shotgun sequence data for five model organisms. Sci Data. 2014;1:140045.

20. Chin CS, Alexander DH, Marks P, Klammer AA, Drake J, Heiner C, Clum A Copeland A, Huddleston J, Eichler EE, et al. Nonhybrid, finished microbial genome assemblies from long-read SMRT sequencing data. Nat Methods. 2013;10:563-9.

21. Chambergo FS, Bonaccorsi ED, Ferreira AJ, Ramos AS, Ferreira Junior JR, Abrahao-Neto J, Farah JP, El-Dorry H. Elucidation of the metabolic fate of glucose in the filamentous fungus Trichoderma reesei using expressed sequence tag (EST) analysis and CDNA microarrays. J Biol Chem. 2002;277:13983-8.

22. Aign V, Schulte U, Hoheisel JD. Hybridization-based mapping of Neurospora crassa linkage groups II and V. Genetics. 2001;157:1015-20.

23. Li W-C, Chuang YC, Chen CL, Wang TF. Hybrid infertility: The dilemma or opportunity of applying sexual development to improve Trichoderma reesei industrial strains. In: Schmoll M, Dattenböck C, editors. Gene expression systems in fungi: advancements and applications. Berlin: Springer International Publishing; 2016. p. 351-9.

24. Ries L, Pullan ST, Delmas S, Malla S, Blythe MJ, Archer DB. Genome-wide transcriptional response of Trichoderma reesei to lignocellulose using RNA sequencing and comparison with Aspergillus niger. BMC Genom. 2013;14:541.

25. Dos Santos Castro L, Pedersoli WR, Antonieto AC, Steindorff AS, SilvaRocha R, Martinez-Rossi NM, Rossi A, Brown NA, Goldman GH, Faca VM, et al. Comparative metabolism of cellulose, sophorose and glucose in Trichoderma reesei using high-throughput genomic and proteomic analyses. Biotechnol Biofuels. 2014;7:41.

26. Li B, Ruotti V, Stewart RM, Thomson JA, Dewey CN. RNA-Seq gene expression estimation with read mapping uncertainty. Bioinformatics. 2010;26:493-500.

27. Wagner GP, Kin K, Lynch VJ. Measurement of mRNA abundance using RNA-seq data: RPKM measure is inconsistent among samples. Theory Biosci (Theorie in den Biowissenschaften). 2012;131:281-5.

28. Schmoll M, Seibel C, Tisch D, Dorrer M, Kubicek CP. A novel class of peptide pheromone precursors in ascomycetous fungi. Mol Microbiol. 2010;77:1483-501.

29. Tachibana C, Yoo JY, Tagne JB, Kacherovsky N, Lee TI, Young ET. Combined global localization analysis and transcriptome data identify genes that are directly coregulated by Adr1 and Cat8. Mol Cell Biol. 2005;25:2138-46
30. Borkovich KA, Alex LA, Yarden O, Freitag M, Turner GE, Read ND, Seiler S, Bell-Pedersen D, Paietta J, Plesofsky N, et al. Lessons from the genome sequence of Neurospora crassa: tracing the path from genomic blueprint to multicellular organism. Microbiol Mol Biol Rev. 2004;68:1-108.

31. Topp CN, Zhong CX, Dawe RK. Centromere-encoded RNAs are integral components of the maize kinetochore. Proc Natl Acad Sci. 2004;101:15986-91.

32. Chan FL, Marshall OJ, Saffery R, Kim BW, Earle E, Choo KH, Wong LH. Active transcription and essential role of RNA polymerase II at the centromere during mitosis. Proc Natl Acad Sci. 2012;109:1979-84.

33. Choi ES, Stralfors A, Castillo AG, Durand-Dubief M, Ekwall K, Allshire RC. Identification of noncoding transcripts from within CENP-A chromatin at fission yeast centromeres. J Biol Chem. 2011;286:23600-7.

34. Gent Jl, Dawe RK. RNA as a structural and regulatory component of the centromere. Annu Rev Genet. 2012;46:443-53.

35. Blat Y, Kleckner N. Cohesins bind to preferential sites along yeast chromosome III, with differential regulation along arms versus the centric region. Cell. 1999;98:249-59.

36. Laloraya S, Guacci V, Koshland D. Chromosomal addresses of the cohesin component Mcd1p. J Cell Biol. 2000;151:1047-56.

37. Glynn EF, Megee PC, Yu HG, Mistrot C, Unal E, Koshland DE, DeRisi JL, Gerton JL. Genome-wide mapping of the cohesin complex in the yeast Saccharomyces cerevisiae. PLoS Biol. 2004;2:E259.

38. Gasser SM. Chromosome structure. Coiling up chromosomes. Curr Biol. 1995;5:357-60

39. Kleckner N. Meiosis: how could it work? Proc Natl Acad Sci. 1996;93:8167-74.

40. Guacci V, Koshland D, Strunnikov A. A direct link between sister chromatid cohesion and chromosome condensation revealed through the analysis of MCD1 in S. cerevisiae. Cell. 1997;91:47-57.

41. Hou C, Li L, Qin ZS, Corces VG. Gene density, transcription, and insulators contribute to the partition of the Drosophila genome into physical domains. Mol Cell. 2012:48:471-84.

42. Cohen BA, Mitra RD, Hughes JD, Church GM. A computational analysis of whole-genome expression data reveals chromosomal domains of gene expression. Nat Genet. 2000;26:183-6.

43. Nora EP, Lajoie BR, Schulz EG, Giorgetti L, Okamoto I, Servant N, Piolot T, van Berkum NL, Meisig J, Sedat J, et al. Spatial partitioning of the regulatory landscape of the $\mathrm{X}$-inactivation centre. Nature. 2012:485:381-5.

44. Cavalli G, Misteli T. Functional implications of genome topology. Nat Struct Mol Biol. 2013;20:290-9.

45. Gibcus JH, Dekker J. The hierarchy of the 3D genome. Mol Cell. 2013;49:773-82.

46. Aramayo R, Selker EU. Neurospora crassa, a model system for epigenetics research. Cold Spring Harb Perspect Biol. 2013;5:a017921.

47. Galazka JM, Klocko AD, Uesaka M, Honda S, Selker EU, Freitag M. Neurospora chromosomes are organized by blocks of importin alpha-dependent heterochromatin that are largely independent of H3K9me3. Genome Res. 2016;26:1069-80.

48. Cambareri EB, Jensen BC, Schabtach E, Selker EU. Repeat-induced G-C to A-T mutations in Neurospora. Science. 1989;244:1571-5.

49. Freitag M, Williams RL, Kothe GO, Selker EU. A cytosine methyltransferase homologue is essential for repeat-induced point mutation in Neurospora crassa. Proc Natl Acad Sci. 2002;99:8802-7.

50. Clutterbuck AJ. Genomic evidence of repeat-induced point mutation (RIP) in filamentous ascomycetes. Fungal Genet Biol. 2011;48:306-26.

51. Horns F, Petit E, Yockteng R, Hood ME. Patterns of repeat-induced point mutation in transposable elements of basidiomycete fungi. Genome Biol Evol. 2012;4:240-7.

52. Wang X, Hsueh YP, Li W, Floyd A, Skalsky R, Heitman J. Sex-induced silencing defends the genome of Cryptococcus neoformans via RNAi. Genes Dev. 2010;24:2566-82.

53. Braumann I, van den Berg M, Kempken F. Repeat induced point mutation in two asexual fungi, Aspergillus niger and Penicillium chrysogenum. Curr Genet. 2008;53:287-97.

54. Chiara M, Fanelli F, Mule G, Logrieco AF, Pesole G, Leslie JF, Horner DS, Toomajian C. Genome sequencing of multiple isolates highlights subtelomeric genomic diversity within Fusarium fujikuroi. Genome Biol Evol. 2015;7:3062-9. 
55. Dhillon B, Gill N, Hamelin RC, Goodwin SB. The landscape of transposable elements in the finished genome of the fungal wheat pathogen Mycosphaerella graminicola. BMC Genom. 2014;15:1132

56. Clutterbuck AJ. MATE transposable elements in Aspergillus nidulans: evidence of repeat-induced point mutation. Fungal Genet Biol. 2004;41:308-16.

57. Schuster A, Bruno KS, Collett JR, Baker SE, Seiboth B, Kubicek CP, Schmoll $M$. A versatile toolkit for high throughput functional genomics with Trichoderma reesei. Biotechnol Biofuels. 2012;5:1.

58. Hane JK, Oliver RP. RIPCAL: a tool for alignment-based analysis of repeatinduced point mutations in fungal genomic sequences. BMC Bioinform. 2008;9:478.

59. Hane JK, Oliver RP. In silico reversal of repeat-induced point mutation (RIP) identifies the origins of repeat families and uncovers obscured duplicated genes. BMC Genom. 2010;11:655.

60. Watters MK, Randall TA, Margolin BS, Selker EU, Stadler DR. Action of repeat-induced point mutation on both strands of a duplex and on tandem duplications of various sizes in Neurospora. Genetics. 1999;153:705-14.

61. Selker EU, Tountas NA, Cross SH, Margolin BS, Murphy JG, Bird AP, Freitag M. The methylated component of the Neurospora crassa genome. Nature. 2003;422:893-7.

62. Galagan JE, Calvo SE, Borkovich KA, Selker EU, Read ND, Jaffe D, FitzHugh W, Ma LJ, Smirnov S, Purcell S, et al. The genome sequence of the filamentous fungus Neurospora crassa. Nature. 2003;422:859-68.

63. Guangtao Z, Hartl L, Schuster A, Polak S, Schmoll M, Wang T, Seid I V, Seiboth B. Gene targeting in a nonhomologous end joining deficient Hypocrea jecorina. J Biotechnol. 2009;139:146-51.

64. Margolin BS, Garrett-Engele PW, Stevens JN, Fritz DY, Garrett-Engele C, Metzenberg RL, Selker EU. A methylated Neurospora 5 S rRNA pseudogene contains a transposable element inactivated by repeat-induced point mutation. Genetics. 1998;149:1787-97.

65. Kim JM, Vanguri S, Boeke JD, Gabriel A, Voytas DF. Transposable elements and genome organization: a comprehensive survey of retrotransposons revealed by the complete Saccharomyces cerevisiae genome sequence. Genome Res. 1998;8:464-78.

66. Jordan IK, McDonald JF. Comparative genomics and evolutionary dynamics of Saccharomyces cerevisiae Ty elements. Genetica. 1999;107:3-13.
67. Bowen NJ, Jordan IK, Epstein JA, Wood V, Levin HL. Retrotransposons and their recognition of pol II promoters: a comprehensive survey of the transposable elements from the complete genome sequence of Schizosaccharomyces pombe. Genome Res. 2003;13:1984-97.

68. Goodwin TJ, Poulter RT. The diversity of retrotransposons in the yeast Cryptococcus neoformans. Yeast. 2001;18:865-80.

69. Labbe J, Murat C, Morin E, Tuskan GA, Le Tacon F, Martin F. Characterization of transposable elements in the ectomycorrhizal fungus Laccaria bicolor. PLoS ONE. 2012;7:e40197.

70. Hakkinen M, Arvas M, Oja M, Aro N, Penttila M, Saloheimo M, Pakula TM. Re-annotation of the CAZy genes of Trichoderma reesei and transcription in the presence of lignocellulosic substrates. Microbial Cell Fact. 2012;11:134.

71. Solovei I, Thanisch K, Feodorova Y. How to rule the nucleus: divide et impera. Curr Opin Cell Biol. 2016:40:47-59.

72. Raffaele S, Kamoun S. Genome evolution in filamentous plant pathogens: why bigger can be better. Nat Rev Microbiol. 2012;10:417-30.

73. Lo Presti L, Lanver D, Schweizer G, Tanaka S, Liang L, Tollot M, Zuccaro A, Reissmann S, Kahmann R. Fungal effectors and plant susceptibility. Annu Rev Plant Biol. 2015;66:513-45.

74. Rouxel T, Grandaubert J, Hane JK, Hoede C, van de Wouw AP, Couloux A, Dominguez V, Anthouard V, Bally P, Bourras S, et al. Effector diversification within compartments of the Leptosphaeria maculans genome affected by repeat-induced point mutations. Nat Commun. 2011;2:202.

75. Soyer JL, El Ghalid M, Glaser N, Ollivier B, Linglin J, Grandaubert J, Balesdent MH, Connolly LR, Freitag M, Rouxel T, et al. Epigenetic control of effector gene expression in the plant pathogenic fungus Leptosphaeria maculans. PLoS Genet. 2014;10:e1004227.

76. Kupfer DM, Drabenstot SD, Buchanan KL, Lai H, Zhu H, Dyer DW, Roe BA, Murphy JW. Introns and splicing elements of five diverse fungi. Eukaryot Cell. 2004;3:1088-100.

77. Liao Y, Smyth GK, Shi W. featureCounts: an efficient general purpose program for assigning sequence reads to genomic features. Bioinformatics. 2014;30:923-30.

78. Steiger MG, Vitikainen M, Uskonen P, Brunner K, Adam G, Pakula T, Penttila M, Saloheimo M, Mach RL, Mach-Aigner AR. Transformation system for Hypocrea jecorina (Trichoderma reesei) that favors homologous integration and employs reusable bidirectionally selectable markers. Appl Environ Microbiol. 2011;77:114-21.

\section{Submit your next manuscript to BioMed Central and we will help you at every step:}

- We accept pre-submission inquiries

- Our selector tool helps you to find the most relevant journal

- We provide round the clock customer support

- Convenient online submission

- Thorough peer review

- Inclusion in PubMed and all major indexing services

- Maximum visibility for your research

Submit your manuscript at www.biomedcentral.com/submit
() Biomed Central 